\title{
Thermodynamics and Structure of Self-assembled Networks
}

\author{
A.G. Zilman* and S.A. Safran \\ Dept. of Materials and Interfaces \\ Weizmann Institute of Science 76100 Rehovot, Israel
}

October 28, 2018

\begin{abstract}
We study a generic model of self-assembling chains which can branch and form networks with branching points (junctions) of arbitrary functionality. The physical realizations include physical gels, wormlike micells, dipolar fluids and microemulsions. The model maps the partition function of a solution of branched, self-assembling, mutually avoiding clusters onto that of a Heisenberg magnet in the mathematical limit of zero spin components. Regarding thermodynamics properties as well as scattering strucuture factor, the mapping accounts rigorously for all possible cluster configuration, except for closed rings. The model is solved in the mean field approximation. It is found that despite the absence of any specific interaction between the chains, the presence of the junctions induces an effective attraction between the monomers, which in the case of three-fold junctions leads to a first order reentrant phase separation between a dilute phase consisting mainly of single chains, and a dense network, or two network phases. The model is then modified in order to predict the structural properties on the mean field level. Independent of the phase separation, we predict the percolation (connectivity) transition at which an infinite network is formed. The percolation transition partially overlaps with the first-order transition. The percolation transition is a
\end{abstract}

*e-mail:anton.zilman@weizmann.ac.il 
continuous, non thermodynamic transition that describes a change in the topology of the system but not a thermodynamic phase transition. Our treatment which predicts both the thermodynamic phase equilibria as well as the spatial correlations in the system allows us to treat both the phase separation and the percolation threshold within the same framework. The density-density correlation correlation has a usual Ornstein-Zernicke form at low monomer densities. At higher densities, a peak emerges in the structure factor, signifying an onset of medium-range order in the system. Implications of the results for different physical systems are discussed.

\section{Introduction}

Networks and branched structures are ubiquitous in both natural and synthetic materials and form under a variety of equilibrium and non-equilibrium conditions. In this paper we present a theory that describes in a unified way the structure and thermodynamic properties of equilibrium networks and their relation to several soft condensed matter systems, such as gels, wormlike micelles, microemulsions, and dipolar liquids and colloids. In all these systems the networks consist of cross-linked elongated objects (e.g., polymer chains in the case of a gel). The study of network phases is of both theoretical and practical interest. From the practical point of view, gels and sol-gel systems are at the core of many industrial, biological and bio-medical applications. Examples range from novel plastics and food processing to gel chromatography and tissue implants [10,26]. Despite intensive theoretical and experimental investigations carried out over the past three decades, many features of network-forming systems still remain unclear. For example, whether the gelation transition in the physical gels is a first-order or a continuous transition, is currently under debate [25, 26, 29, 36]. Experiments have not yet provided a clear-cut answer, because the gelation transition can be obscured by the van der Waals interaction [35]. Another example is the transition of a solution of worm-like micelles to a self-assembled network that has been suggested to occur on the basis of rheological measurements; this has also been discussed theoretically [32, 33]. Network formation may also be responsible for the 'closed loop' phase diagrams of dipolar and magnetic liquids and colloids and of microemulsions 45. Cryo-electron microscopy shows clear evidence of coexisting network phases in dilute microemulsions [34]. In many of the systems mentioned above, the chains themselves are self-assembled in 
an equilibrium manner from a large number of monomers.

We focus here on the thermodynamic behavior and structure of systems with thermoreversible cross-linking; this means that the cross-links can break and reform under the influence of thermal fluctuations. It turns out however, that many of the large scale structural properties of network-forming phases are independent of the precise nature of the cross-links. In many of the complex systems mentioned above, an understanding of the local and large scale correlations are also of importance. Certain structural transitions cannot be detected by examining the thermodynamic properties alone, but are expressed by the nature of the correlations between different components of the system. One example is the percolation transition, at which a network spanning the whole system is formed, as schematically shown in Fig. 3. It is a continuous transition, unrelated to the thermodynamic properties of the system. Generally speaking, one would like to know both the thermodynamic behavior of these systems as well as such experimentally measurable quantities as density-density correlation functions and response functions to applied fields such as an electromagnetic field or hydrodynamic flow.

To answer these questions, at least within a mean-field approach, we study a generic system consisting of self-assembled chains which can branch and form networks. Each chain consists of a large number of 'monomers'. The chains are self- and mutually- avoiding but are permitted to branch (crosslink). We shall call both the branching points and the cross-links 'junctions'. The exact physical interpretation of the 'monomers' and 'junctions' will differ form system to system. In each particular case, the energy and other properties of the junctions and the ends can be calculated from microscopic considerations, e.g., molecular packing for surfactant systems, or dipolar interaction energies applicable to ferrofluids.

We present here a lattice model which establishes the equivalence between a solution of branched self-avoiding self-assembling chains and a Heisenberg magnet in the limit of 'zero' spin components; we show that their partition functions can be mapped onto another. This model, known as ' $n=0$ ' model, was proposed by De Gennes to study polymer solutions and we have modified it in order to include the possibility of branching. Regarding the thermodynamic properties, including the density fluctuations, the model is exact and accounts for all possible configurations of equilibrium branched clusters (excluding separate closed rings, whose influence is or importance only at very low densities). This formulation of the problem enables us to explore both thermodynamic and structural properties of self-assembling branching 
chains in a unified manner. The model has been treated in the mean field approximation and our major conclusions are summarized in Fig. 1, which shows the phase diagram of the system as a function of the density and temperature. We find that despite the absence of any specific interactions between the monomers, the presence of the junctions induces an effective attraction between the monomers. The model can be amended in order to study the topological properties of the system and correlate them with the thermodynamic behavior. As the monomer density is increased or the temperature decreased, the system passes through a percolation (connectivity) threshold, where a network spanning the entire volume is formed. This transition is purely topological, and has no thermodynamic signature. However, the junction-induced attraction does modify the concentration of monomers at which the percolation threshold occurs; as the temperature is decreased, the threshold is decreased compared with its value in the limit of infinite temperature, where the interactions are irrelevant.

For three-fold junctions, the junction-induced attraction is strong enough to drive a first-order phase separation, where the system separates into a low-density and a high density phases. This transition is of purely entropic origin, because there are no any specific interactions between the monomers. The physical reason for the transition is the higher entropy of the junctionsdominated high density phase: although the translational entropy of the chains is lower in the high density phase, it is overcompensated by the increase of the entropy of the self-assembled junctions, abundant in the highdensity phase. In this respect, this entropy-induced transition is similar to the crystallization of rigid spheres and the isotropic-nematic transition. The transition line terminates at a critical point. For four-fold and higher functionality junctions, the junction induced transition is too weak to drive a phase separation. In this case, the junction-induced interaction merely renormalizes the excluded volume interaction between the chains, and drives the system closer to the $\Theta$-point. The structural percolation transition, of course, is also present above the critical temperature of the first-order phase separation. At very low temperatures and monomer densities, the chains are depolymerized and the system consists mainly of separate monomers.

The nature of the phases in equilibrium depends on the temperature, but also on the rigidity of the chains and details of the junction configurations. In general, there are three possibilities: (i) a phase of dilute chains that coexists with a connected network, (ii) two coexisting networks, or (iii) the coexistence of dilute and dense phases of disjointed, branched aggregates, 
although not predicted by our model, is also possible.

Treatment of the spatial density variations within the mean field theory shows that the density-density correlation function, relevant for scattering experiments, has a simple Ornstein-Zernicke form for relatively low densities of the self-assembling monomers. For higher densities, a peak emerges in the structure factor as a function of the scattering wavevector; this indicates the emergence of medium range (longer range than the lattice size but not long range order) correlations in the system. In the intermediate regime the structure factor is a monotonically increasing function of the wavevector. This is expected in any dense system: at high densities, the system has a low compressibility at long wavelengths and this suppresses the small wavevector scattering. The absence of a peak in the structure factor does not imply the absence of the network: there is a region in the phase diagram beyond the percolation line ( where an infinite network exists) where there is indeed no peak in the density-density correlation function. The precise location and strength of the peak depends on the monomer density and the number of junctions present. As discussed in Section. [7, these theoretical predictions may be related to the scattering peak observed experimentally in bicontinuous microemulsions.

The predictions of the thermodynamic and structural properties can be put in the context of previous work on the physics of 'living' (self-assembling) polymers and networks; this area has been the focus of extensive experimental and theoretical attention over the past two decades. Most notably, phenomenological Flory type (chains with linkers) theories have been employed to model physical gels [29, 36]. Another important contribution is the work of Drye and Cates that was motivated by studies of worm like micelles 41. However, the predictions of these various studies regarding the properties of the gelation transition vary depending on the details of the models that are studied, the assumptions regarding the solvent quality and other parameters. This is partially due to the fact that these studies [29, 36] did not emphasize the extreme sensitivity of the gelation transition to the functionality of the junctions and the importance of the competition of the branching points and the free ends. These previous, more heuristic, mean-field theories could not be extended in a simple manner to predict the correlations.

We have therefore focused on the more rigorous ' $n=0$ ' model, which allows one to evaluate exactly the number of all possible configurations of branched self- and mutually avoiding chains on a lattice. Lattice approximation models the configurations of the chains only approximately at short 
lenghtscales. However, as long as one is interested the behavior of the system on lengthscales much larger than the lattice constant, (i.e., thermodynamics) the choice of the lattice does not influence any of the qualitative predictions of the model. The ' $n=0$ ' model has been successfully used to explain the properties of associating monomers and has been applied to the polymerization of sulfur [20] and worm like micellar solutions [17]. The extension of the model to $n=1$ was used to model systems consisting of chains and rings in equilibrium [21]. Our model is similar to the one employed by Isaacson an Lubensky 23 to which it can be related (cf. Appendix D). These authors studied the continuum version of the ' $n=0$ ' model with a cubic term in the Hamiltonian in the context of percolation focusing on the scaling behavior of the model to study the properties of the gelation-percolation transition but did not calculate the thermodynamic transitions (such as the phase separation predicted here) or study the monomer density fluctuations and scattering structure factor. In our model, however, the junctions are not modelled as point-like objects, and the treatment of topological structure is simpler than of Ref. [23], but is applicable only in the mean field approximation. The $n=1$ model, which accounts also for closed rings, but does not properly take into account the self-avoidance, was used by Lequeux, Elleuch and Pfeuty 32 to study general properties of micellar solutions without considering the topology of the network. Similarly, in the two-field model of Ref. 31], correlations and structure were not studied and no analytic expression for the free energy was obtained.

This paper is organized as follows. In Sec. 2.1 we review the ' $n=0$ ' model without junctions. In Sec. 2.2 we extend the model to include the possibility of junctions and establish the correspondence between this model and the physics of the problem of self-assembled networks. In Sec. 3 we calculate the free energy and phase diagram in the mean-field approximation. We next extend the mean field theory to include spatial variations of the monomer density and in Sec.⿴囗十, predict the spatial variations and correlations. In the Sec. 5 we show how the model can be modified on the mean-field level to enable us to predict the topological properties and correlate them with the thermodynamic behavior. The approximation employed, is of the same level as the classical Flory-Stockmayer theories of gelation. In particular, in Sec. 5.2 we discuss the structure and evolution of a single branched cluster as a function of monomer density and the temperature, and the emergence of a connected network. Sec. 6 describes the extension of our model and its predictions to the case of rigid and semiflexible chains. In Sec. 7 we 
Figure 1: Phase diagram of solution of self-assembling branched chains as a function of temperature $T$ in units of the end energy $\epsilon_{e}$, and monomer volume frcation $\phi$. The junction energy has been chosen to be $\epsilon_{j}=\epsilon_{e} / 4$. The thick line shows the phase separation region. The dashed line is the percolation line. To the left of this line the system consisits a solution of disconnected (but possibly entangled) branched chains; to the right there is an infinite connected network. Moving along the line $b$, the actual gelation transition will be seen as a continuous non-thermodynamic transition, while moving along $a$ will result in the first-order thermodynamic transition. Thus, coexistence of two connected networks is possible at high enough temperatures.

discuss the results and their application to particular physical systems such as physical gels, microemulsions, dipolar fluids and wormlike micelles.

\section{Zero-component' Heisenberg model}

\subsection{Self-avoiding chains.}

For systems with no junctions, the ' $n=0$ ' model has been extensively studied in the context of polymer solutions and micellar systems [2, 12, [17, 19]. For the sake of completeness we review here the derivation of the model; in Sec. 2.2 we extend the model to include junctions. Readers familiar with 
' $n=0$ ' model can proceed directly to Sec. 2.2. We consider a system that comprises a solvent and a collection of self-assembling chains, each consisting of a large number of identical monomer units. The physical nature of the 'monomers' can be different. For example, in physical gels these would be the individual molecular units that comprise the polymers, in microemulsions these would be surfactant covered oil (or water) domains and in dipolar

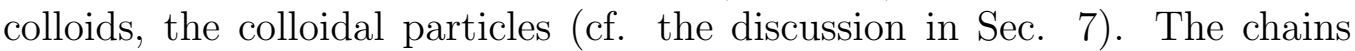
are self- and mutually avoiding, that is, they cannot intersect themselves and each other. We focus on self-assembling systems where the monomers are relatively weakly associated within a chain; thus, individual monomers can be freely exchanged between the chains. In this case, the equilibrium distribution of chain lengths is not fixed, but is determined by the values of relevant physical parameters, as we discuss later. We will consider the system in the grand-canonical ensemble, in equilibrium with the bath of monomers of the chemical potential $\mu$. We assume that the energy of a monomer at the end of the chain, $\epsilon_{e}$, relative to that of a monomer in the middle of the chain is positive, $\epsilon_{e}>0$. This is indeed the case for micellar systems and microemulsions, because (in the regime where long chains are formed) the bending energy of the surfactant layer is lower in the cylindrical part than in the semi-spherical end-caps [ [] ]. The same is true for dipolar particles, because the electrostatic energy of a dipole in the middle of the chain with two neighbors, is lower than that of a dipole at the chain end, where it has only one neighbor dipole. If $\epsilon_{e}<0$, the self-assembling chains will be very short and the solution will mostly consist of individual monomers; this is not the limit that we are interested in. The end energy, taken with the opposite sign, $-\epsilon_{e}$, can also be interpreted as $\mu_{p}$, the chemical potential conjugate to the number of chains in the solution, $N_{p}$. This interpretation is usually adopted in discussion of regular polymer solutions [2, 12]. For the systems of interest, the energy $\epsilon_{e}$ is determined from microscopic considerations, be it molecular packing or electrostatics. The number of chains and average molecular weight are not fixed but determined by physically controllable parameters, namely, the temperature, the total monomer density $\phi$ and $\epsilon_{e}$. For simplicity we imagine that the monomers and the solvent molecules occupy the sites of a $d$-dimensional lattice. With the above notation, the grand canonical partition function of a solution of equilibrium chains is given by

$$
Z=\sum_{\left\{N, N_{e}\right\}} \exp [\mu N / T] \exp \left[-\epsilon_{e} N_{e} / T\right] \mathcal{N}\left(N, N_{e}\right)
$$


where $N$ is the number of monomers in a given realization, $N_{e}$ is the total number of free ends, and $\mathcal{N}\left(N, N_{e}\right)$ is the number of ways to arrange $N_{e} / 2$ chains of total length $N$ on a lattice. Thermodynamic quantities can be obtained from Eq.(1) by differentiation with respect to the relevant parameter. For example, the mean number of monomers and chains, $\bar{N}$ and $\bar{N}_{p}$ respectively, are:

$$
\begin{aligned}
\bar{N} & =\frac{\partial \ln Z}{\partial \mu} \\
\bar{N}_{p} & =\frac{\partial \ln Z}{\partial \epsilon_{e}}
\end{aligned}
$$

The direct combinatorial calculation of $\mathcal{N}\left(N, N_{e}\right)$ is a formidable task. A way of circumventing the difficulties involved in its calculation, was proposed by De Gennes 13. It was later refined by equivalent methods by different authors 14, 15]. The calculation of $\mathcal{N}\left(N, N_{e}\right)$ is done by relating the partition function of the Eq.(11) to that of a certain spin model on a lattice. Consider the $n$-component Heisenberg model on a $d$-dimensional lattice whose Hamiltonian is given by

$$
H=-\sum_{\left\{\vec{S}_{i}\right\}} J \vec{S}_{i} \cdot \vec{S}_{j}-\sum_{\left\{\vec{S}_{j}\right\}} \vec{h}_{i} \cdot \vec{S}_{i}
$$

where the summation is over all distinct pairs of nearest neighbors $i, j$. Spin $\vec{S}_{i}$ is an $n$-component vector normalized at each site as

$$
\left|\vec{S}_{i}\right|^{2}=\sum_{\alpha=1}^{n} S_{i, \alpha}^{2}=n
$$

where $\alpha$ indexes the components of $\vec{S}_{i}$. We arbitrarily choose the field $\vec{h}$ to point in the direction $(1,0, \ldots, 0)$.

The partition function corresponding to this spin Hamiltonian is

$$
Z_{H}=\operatorname{Tr} \exp \left[-H / T^{\star}\right]
$$

where the trace operator $\operatorname{Tr}$ signifies an integration over all possible directions of $\vec{S}_{i}$, divided by a normalization, so that the trace $\operatorname{Tr} A$ of any quantity $A$ is:

$$
\operatorname{Tr} A=\frac{\int \prod_{i} d \Omega_{i}^{(n)} A}{\int \prod_{i} d \Omega_{i}^{(n)}}
$$


where $\Omega^{(n)}$ is the $n$-dimensional solid angle. The 'temperature' $T^{\star}$ has no physical meaning and is not related to the real physical temperature of the system of in Eq.(11); we put $T^{\star}=1$ (another choice of $T^{\star}$ would amount to a renormalization of the constants $J$ and $h$ ). Expanding the exponential in $Z_{H}$ of Eq.(3) in a power series, we obtain

$$
Z_{H}=\sum_{k} \frac{1}{k !} \operatorname{Tr} \prod_{\langle i, j\rangle}\left(J \vec{S}_{i} \vec{S}_{j}+h S_{i, 1}\right)^{k}
$$

Each term in Eq.(4) has the following form

$$
\operatorname{Tr}[J^{m} \underbrace{S_{i} S_{j} \ldots S_{i^{\prime}} S_{j^{\prime}}}_{m \text { times }} h^{n} \underbrace{S_{p, 1} \ldots S_{p^{\prime}, 1}}_{n \text { times }}]
$$

Note that in the tracing operation in Eq.(5), all sites are decoupled.

In any realistic system in which the spin $\vec{S}$ with $n$ components is to have a physical interpretation, $n \geq 1$ is required; $n=1$ corresponds to the usual Ising model. However, quantities such as partition function and the cumulant expansion remain mathematically meaningful even when $n<1$. In particular, one can consider the mathematical limit $n \rightarrow 0$ [1, 12]. It can be shown that when $n \rightarrow 0$ (cf. Appendix 8.1),

$$
\operatorname{Tr} S_{i, \alpha} S_{j, \beta}=\delta_{\alpha \beta} \delta_{i j}
$$

and all other cumulants are zero. The spin $\vec{S}$ does not have a physical interpretation in the $n \rightarrow 0$ limit and should be regarded purely as a convenient mathematical device. Precise mathematical meaning can be given to Eq.(6) (cf. Appendix 8.1). In particular, Eq.(6) means that in the expansion (5) the only non-vanishing terms are those in which every spin $S_{i}$ appears twice. As can be seen from Eq. (41), this condition is satisfied by terms consisting of the chains of neighboring bonds: ...J $S_{i} S_{j} J S_{j} S_{j^{\prime}} \ldots$. Since every spin must appear twice for the term to be non-zero, the uncoupled spins must be paired off with another spin at the same site either by (i) closing a chain on itself or by (ii) the appearance of the single-spin, 'field' term $h_{1} S_{1}$. However, in the limit of $n \rightarrow 0$, the terms given by (i) vanish, due to summation over all the components (via summation over the index $\alpha$ ); this sum is proportional to the number of components, $n$, which is equal to zero. The terms that contain the field variables, $h_{1}$, do not vanish because the $h$-terms single out 
one component $\alpha$, parallel to the field $h$. It is easy to see that such terms consist of products of the form

$$
\operatorname{Tr} h_{i, 1} S_{i, 1} J S_{i, 1} S_{j, 1} J S_{j, 1} S_{k, 1} \ldots J S_{q, 1} S_{r, 1} h_{j, 1} S_{r, 1}
$$

that repeat themselves with different $i$ and $r$. Any term of the form of Eq.(7) corresponds to a self-avoiding random walk on a lattice, starting at site $i$ and ending at site $r$. In other words, a term containing the factor $J^{m} h^{2 k}$ counts all the possible configurations of $k$ random walks of total bond length $m$. Thus, the partition function can be written:

$$
Z_{H}=\sum_{\left\{N_{b}, N_{e}\right\}} J^{N_{b}} h^{N_{e}} \mathcal{N}\left(N_{b}, N_{e}\right)
$$

where $\mathcal{N}\left(N, N_{e}\right)$ is the number of ways to arrange on a lattice, an ensemble of self-avoiding random walks with a total number of bonds $N_{b}$ and a total number of ends $N_{e}$. Noting that the number of chains is $N_{p}=N_{e} / 2$ and the number of monomers is $N=N_{b}+N_{p}$, one can see that Eq.(8) is identical to Eq.(11) if the following identification is made

$$
\begin{aligned}
J & =e^{\mu} \\
h & =e^{-\epsilon_{e}} J^{1 / 2} \equiv h_{0} J^{1 / 2}
\end{aligned}
$$

Each term in the sum in Eq.(8) corresponds to a different realization of the grand canonical ensemble of a solution of self-avoiding chains. We have thus demonstrated that the grand canonical partition function of a solution of polydisperse, living polymers is identical to that of the $n$-component Heisenberg model where the number of components $n \rightarrow 0$. As mentioned in the Introduction, the lattice approximation is adequate as long as the chains are much longer than the lattice constant. This theory describes the ensemble of self-assembling 'living' chains, which we are of interest to us. The molecular weight and the length distribution are not fixed but are a functions of external parameters, such as the monomer density. However, this model has also been successfully applied to non-self-assembling polymer solutions (where the degree of polymerization is constant and fixed by the chemical preparation technique). The continuum version of this model is the basis for the application of field-theoretical methods to polymer physics [14, 15, 38]. The self-assembling theory can be used to predict the properties of non-selfassembling polymer solutions of long chains (small $h$ in our formulation), because the scaling behavior of such systems is universal and independent of the detailed chain properties. 


\section{$2.2 \quad$ Three-fold junctions}

The model presented in the previous section can be modified to include the possibility of junctions that connect several chains; this allows a formation of a branched structure and is achieved by introducing an additional term to the Hamiltonian of Eq.(2). Again, we consider the grand canonical partition function of an equilibrium solution of branched cross-linked polymers with reversible cross-links. The cross-links (branching points) are in thermal equilibrium and can break and reform. Similar to the solution of polymer chains discussed above, the grand canonical partition function of the system is

$$
Z_{3}=\sum_{\left\{N, N_{e}, N_{j}\right\}} \exp [\mu N / T] \exp \left[-\epsilon_{e} N_{e} / T\right] \exp \left[-\epsilon_{j} N_{j} / T\right] \mathcal{N}\left(N, N_{e}, N_{j}\right)
$$

where $\epsilon_{j}$ is the energy of a cross-link relative to the energy of the monomer in the middle of a chain, $N_{j}$ is the number of junctions (cross-links) and $N\left(N, N_{e}, N_{j}\right)$ is the number of ways to put self-avoiding branched chains of total length $N$ on a lattice so that $N_{e}$ ends and $N_{j}$ junctions are formed. The chemical potential for the monomers, $\mu$ and the end energy $\epsilon_{e}$ are defined as in the previous section. Similar to the discussion of the end energy and its associated chemical potential, the junction energy taken with the opposite sign, $-\epsilon_{j}$, can be interpreted as the chemical potential conjugate to the number of junctions. We suppose that the physically controllable parameters are the monomer density $\phi$, the temperature $T$ and the ends/junctions energies $\epsilon_{e}, \epsilon_{j}$. In this formulation, the density of ends $\phi_{e}$ and the density of junctions $\phi_{j}$ are not fixed but determined in thermal equilibrium as functions of $\phi$ and $T$.

In this section we show how the three-fold junctions can be described by ' $n=0$ ' model. The generalization to junctions of arbitrary functionality is discussed in the next section. As in the previous section, one can relate the partition function of Eq.(9) to an equivalent spin model on a lattice. The mapping accounts rigorously for all possible configurations of branched self-avoiding clusters, except for closed rings. The influence of rings on the properties of living polymers has been studied in Ref. [21]. However, in the case of branched chains, the influence of closed rings on thermodynamic properties of the system is small, except at very low densities, and they are not treated in this paper. The reason for this is that for any closed ring there is an exponentially big number of branched clusters formed by 
attaching side chains to it. No topological information regarding, e.g., the network formation, or the cluster size distribution can be extracted from the model formulated below. However, it can be modified to include the treatment of topological properties too, as shown in Sec.5.

We introduce the following Hamiltonian that contains a term which couples three adjacent spins:

$$
H_{3}=\sum_{i} \vec{h} \cdot \vec{S}_{i}+\sum_{i j} J\left(\vec{S}_{i} \cdot \vec{S}_{j}\right)+\sum_{i j k} K S_{1, i} S_{1, j} S_{1, k}
$$

where $i, j, k$ sum is over all distinct triplets on the lattice. The field $h$ is chosen to point in the direction $(1,0 \ldots 0)$. The partition function corresponding to this Hamiltonian is

$$
Z_{H_{3}}=\operatorname{Tr}_{\left\{S_{i}\right\}} \exp \left[-H_{3}\left\{S_{i}\right\} / T^{\star}\right]
$$

The 'temperature' $T^{\star}$ can be taken equal to unity and any other choice would amount to a redefinition of the constants $J, K$ and $h$. Proceeding as in the previous section, we expand $Z_{H_{3}}$ in series in powers of $K, J$ and $h$. In the limit $n \rightarrow 0$ the terms in the expansion of $Z_{H_{3}}$ in powers of $J, K$ and $h$ are similar to those discussed in the previous section. However, there are additional terms obtained by inserting into any term of the expansion of Eq.(5) combinations of the form

$$
\ldots J S_{j^{\prime}, 1} S_{j, 1}[\underbrace{K S_{j, 1} S_{m, 1} S_{n, 1}}_{\text {junction }}] J S_{n, 1} S_{n^{\prime}, 1} J S_{m, 1} S_{m^{\prime}, 1} \ldots
$$

Each insertion of this kind corresponds to a three-fold junction on a lattice, joining the sites $j, m, n$ (cf. Fig. Q). Thus, a general term in the expansion of $Z_{H_{3}}$ might look like

$$
\operatorname{Tr} h S_{i, 1} J S_{i, 1} S_{j, 1} \underbrace{\left[K S_{j, 1} S_{n, 1} S_{k, 1}\right]}_{\text {junction }} J S_{n, 1} S_{n^{\prime}, 1} h S_{n^{\prime}, 1} \ldots J S_{k, 1} S_{m, 1} h S_{m, 1}
$$

This particular term, for example, corresponds to a three-fold branched, selfavoiding chain, which has a junction between the points $j, n, k$, and free ends at points $i, n^{\prime}$ and $m$. In other words, the partition function is 


$$
Z_{H_{3}}=\operatorname{Tr} \exp \left[-H_{3}\right]=\sum_{\left\{N_{b}, N_{e}, N_{j}\right\}} J^{N_{b}} h^{N_{e}} K^{N_{j}} \mathcal{N}\left(N_{b}, N_{e}, N_{j}\right)
$$

where $\mathcal{N}\left(N_{b}, N_{e}, N_{j}\right)$ is the number of ways to arrange on a lattice an ensemble of self-avoiding branched random walks with a total number of bonds $N_{b}$ (excluding three 'ghost' bonds at each junctions, cf. Fig. 2), a total number of ends $N_{e}$, and a total number of junctions $N_{j}$. The closed rings fall out form the expansion 14 due to summation over spin components. Each ring produces a contribution proportional to $n$ which tends to zero as $n \rightarrow 0$, analogously to the case of linear chains. From simple geometric considerations, the number of monomers, $N$, is always $N=N_{b}+\frac{1}{2} N_{e}+\frac{3}{2} N_{j}$ (we do not count the junctions as monomers; see Appendix 8.2), regardless of the presence or absence of loops. Note that this formulation does not treat the junctions as point-like objects. Choosing different forms of three-spin coupling in Eq.(10) one can study the influence of the junction size on the system properties (cf. Appendix 8.2). If the following identifications are made

$$
\begin{aligned}
J & =e^{\mu / T} \\
h & =e^{-\epsilon_{e} / T} J^{\frac{1}{2}} \equiv h_{0} J^{\frac{1}{2}} \\
K & =e^{-\epsilon_{j} / T} J^{\frac{3}{2}} \equiv K_{0} J^{\frac{3}{2}}
\end{aligned}
$$

then the $Z_{H_{3}}$ of Eq.(14) becomes identical to $Z_{3}$ of Eq.(9). To summarize, we have shown that in the $n \rightarrow 0$ limit, the grand-canonical ensemble of a solution of branched, reversibly cross-linked polymers is equivalent to a Heisenberg magnet with three-spin term. It is important to emphasize that although the closed rings ( linear, unbranched, chains closed to form a ring) are not included in the expansion (13), all the intracluster loops are counted properly.

The concentrations of the monomers, ends and junctions, $\phi, \phi_{e}$ and $\phi_{j}$ respectively can be obtained by differentiating the partition function with respect to a relevant parameters as follows from Eqs.(9, 14):

$$
\begin{aligned}
\phi & =\frac{1}{V} \frac{\partial \ln Z}{\partial \ln J} ; \quad \phi_{\text {bonds }}=\frac{1}{V} \frac{\partial_{s} \ln Z}{\partial_{s} \ln J} \\
\phi_{e} & =\frac{1}{V} \frac{\partial \ln Z}{\partial \ln h} ; \phi_{j}=\frac{1}{V} \frac{\partial \ln Z}{\partial \ln K}
\end{aligned}
$$


where $\partial_{s}$ denotes a derivative with respect to $J$ with the generalized fugacities $K, J$ and $h$ taken to be independent. The great advantage of the present formulation is that it allows to apply the methods of statistical mechanics developed in the past four decades for the treatment of the spin models.

In some systems, junctions may form spontaneously; in that case, the number of junctions, distribution of the chain intervals between the junctions and the branched clusters' size will be determined by the junction energy $\epsilon_{j}$, the monomer density $\phi$ and the temperature $T$. This is what one expects, for example, in dipolar fluids or microemulsions. In other systems, junction formation may only be possible if linker molecules, that connect several chains, are added to the system. In this case, the grand canonical formulation presented here amounts to the assumption that the system is in equilibrium with a bath of linker molecules with chemical potential $-\epsilon_{j}$.

The grand canonical potential per unit volume, $\omega\left(\epsilon_{j}, \epsilon_{e}, \mu\right)$, is given by

$$
\omega\left(\epsilon_{j}, \epsilon_{e}, \mu\right) / T=-\frac{1}{V} \ln Z\left(K_{0}, h_{0}, J\right)
$$

Other physically realizable situations are related to Eq.(17) by a Legendre transform. For instance, the free energy corresponding to the case where junctions form only in the presence of linker molecules is

$$
g\left(\mu, \epsilon_{e}, N_{j}\right)=\omega-\phi_{j} \ln K_{0}
$$

Of course, in the thermodynamic limit $(V \rightarrow \infty)$ the properties of the system are identical in either ensemble. Here, we will focus on the case where the physically controllable parameters are the total monomer density, $\phi$, and the defect energies, $\epsilon_{e} / T=-\ln \left(h_{0}\right)$ and $\epsilon_{j} / T=-\ln \left(K_{0}\right)$. The corresponding Helmholtz free energy per unit volume is

$$
f=\omega+\mu \phi=\omega+\phi \ln J
$$

\section{Mean field theory}

\subsection{Mean field approximation}

The first approximation in evaluating the free energy is to disregard the spatial variations of the densities and the long range correlations between the 
Figure 2: Junction can be formed between three adjacent sites $i, j, k$. The Boltzmann factor for a junction is $K_{0}=e^{-\epsilon_{j} / T}$.

monomers. In this section we assume that the system is spatially uniform and calculate the free energy as a function of the average monomer volume fraction $\phi$, and the ends and junction densities, $\phi_{e}$ and $\phi_{j}$, respectively. A mean-field calculation of the free energy for a system with no junctions has been presented in Refs. [19, 20]. We extend this calculation by an alternative method to a system that contains both ends and junctions. We first calculate the grand canonical potential $\Omega / T=-\ln Z$ in the mean field approximation, which is generally known to predict the qualitative thermodynamic behavior correctly 8$]$. As we shall show subsequently in Sec. 5, the mean field approximation neglects the internal loops in the branched clusters, because only local properties are preserved in the mean field approximation. Geometrically, it is equivalent to Flory construction [⿴囗十 when each cluster is constructed by adding consecutive bonds, disregarding the positions of the previously placed ones, and the long range correlations are lost. In particular, intra-cluster self-avoidance is neglected in the mean field approximation. However, the excluded volume between different clusters is taken into account. The partition function $Z$ is given by

$$
Z=\operatorname{Tr} \exp \left[-\sum_{i, j} J \vec{S}_{i} \cdot \vec{S}_{j}-\sum_{i, j, k} K S_{1, i} S_{1, j} S_{1, k}-\sum_{i} \vec{h} \cdot \vec{S}\right]
$$


The sums in the argument of the exponent of Eq.(18) are over distinct pairs and triples, as explained in Sec. 2.2. We now note the following identity

$$
\vec{S}_{i} \vec{S}_{j}=\underline{\left(\vec{S}_{i}-\vec{S}\right)\left(\vec{S}_{j}-\vec{S}\right)}-\vec{S}^{2}+\vec{S}\left(\vec{S}_{i}+\vec{S}_{j}\right) \simeq-\vec{S}^{2}+\vec{S}\left(\vec{S}_{i}+\vec{S}_{j}\right)
$$

where $\vec{S}=\left\langle\vec{S}_{i}\right\rangle$. The underlined term in the equation is quadratic in the deviation of the local spin from its average value $S$. It is this term that is neglected in the mean field approximation. As the 'magnetic field' $h$ has been chosen to point in the direction $(1,0 . .0)$ the only non-zero component of the average spin is $S_{i, 1}$. Therefore, the transverse components of the spin $\vec{S}$ do not contribute to the mean field approximation, due to the fact that $\left\langle S_{i, \perp}\right\rangle=0$. So, in the following we drop the vector sign and $S_{i}$ signifies the component of $\vec{S}_{i}$ in the direction of $h$. Similarly, the mean field approximation for the three-spin term is:

$$
S_{i} S_{j} S_{k} \simeq-2 S^{3}+S\left(S_{i}+S_{j}+S_{k}\right)
$$

Thus, the mean field approximation to the Hamiltonian reads

$$
H_{\mathrm{MF}}=V\left(\frac{1}{2} q J S^{2}+2 \alpha K S^{3}\right)-\sum_{i}\left(q J S+3 \alpha K S^{2}+h\right) S_{i}
$$

where $V$ is the number of lattice sites (which tends to infinity in the thermodynamic limit) and the sum is over all lattice sites; $q$ is the lattice coordination number. The prefactor $\alpha=\frac{1}{3} \alpha^{\prime}$, where $\alpha^{\prime}$ is the number of possible configurations of $j, k$ in the triple term $i, j, k$ making a junction around the cite $i$. The exact value of $\alpha^{\prime}$ depends on the type of the lattice used and on the physical assumptions made in counting the physically relevant junction configurations. Physically, it represents the entropy associated with local rearrangements of a single junction and we discuss its value for semi-flexible or rigid systems in Sec.6. In most of this paper, we shall use the value of $\alpha^{\prime}$ appropriate for a simple cubic lattice $\alpha^{\prime}=q(q-2) / 3$ (cf. Appendix 8.2). A particular feature of the $n \rightarrow 0$ limit, following from Eq.(6) [1, 12, 19] (cf. also Appendix 8.1) is the fact that for an arbitrary vector $\vec{k}$,

$$
\lim _{n \rightarrow 0} \operatorname{Tr} e^{\vec{k} \vec{S}_{i}}=1+\frac{1}{2} k^{2}
$$


Consequently, performing the trace in Eq.(18), we get

$$
\frac{1}{V}(\Omega / T) \equiv \omega=\frac{1}{2} q J S^{2}+2 \alpha K S^{3}-\ln \left(1+\frac{1}{2}\left(q J S+3 \alpha K S^{2}+h\right)^{2}\right)
$$

The average value of the spin, $S$, is given by

$$
S=-\frac{\partial \omega}{\partial h}=\frac{1}{V}\left\langle\sum_{i} S_{i}\right\rangle
$$

The total monomer density is

$$
\phi=\phi_{b o n d s}+\frac{1}{2} \phi_{e}+\frac{3}{2} \phi_{j}=-\frac{\partial_{s} \omega}{\partial_{s} \ln J}-\frac{1}{2} \frac{\partial \omega}{\partial \ln h}-\frac{3}{2} \frac{\partial \omega}{\partial \ln K}=-\frac{\partial \omega}{\partial \ln J}
$$

as follows from Eq.(16), and can be also understood by a simple geometrical argument (cf. Appendix 8.2). Finally, combining all the equations we find:

$$
\begin{aligned}
\omega & =\frac{1}{2} q J S^{2}+2 \alpha K S^{3}-\ln \left(1+\frac{1}{2}\left(q J S+3 \alpha K S^{2}+h\right)^{2}\right) \\
S & =-\frac{\partial \omega}{\partial h}=\frac{q J S+3 \alpha K S^{2}+h}{1+\frac{1}{2}\left(q J S+3 \alpha K S^{2}+h\right)^{2}} \\
\phi & =\frac{1}{2} S(\underbrace{q J S+3 \alpha K S^{2}+h}_{x})
\end{aligned}
$$

Denoting $q J S+3 \alpha K S^{2}+h$ by $x$, the solution of this system of equations gives

$$
\begin{aligned}
\phi & =\frac{1}{2} S x \\
S & =\frac{x}{1+\phi x / S}
\end{aligned}
$$

Consequently

$$
\begin{aligned}
x & =\sqrt{\frac{2 \phi}{1-\phi}} ; \quad 1+\frac{1}{2} x^{2}=\frac{1}{1-\phi} ; S=\sqrt{2 \phi(1-\phi)} \\
\omega & =q J \phi(1-\phi)+2 \alpha K(2 \phi)^{3 / 2}(1-\phi)^{3 / 2}+\ln (1-\phi)
\end{aligned}
$$


It follows that in the limit of systems with a relatively small number of junctions and ends, compared to the total number of monomers in the chains, $\left(h_{0}(2 \phi)^{1 / 2} \ll \phi, K_{0}(2 \phi)^{3 / 2} \ll \phi\right)$,

$$
q J=\left(x-h-3 \alpha K S^{2}\right) / S \simeq \frac{1}{1-\phi}\left(1-\frac{h_{0}}{\sqrt{2 \phi}}-3 \alpha K_{0} \sqrt{2 \phi}\right)
$$

Because we are interested in those systems where the density, $\phi$, (and not the Lagrange multiplier, $J$, ) is a physically controllable parameter, we perform a Legendre transform $f=\omega+\phi \ln q J$ in order to obtain the Helmholtz free energy per unit volume $f\left(\phi, h_{0}, K_{0}\right)$. Up to the first order in $h_{0}$ and $K_{0}$,we find:

$$
f / T \simeq(1-\phi)(\ln (1-\phi)-1)-\frac{h_{0}(2 \phi)^{1 / 2}}{q^{1 / 2}}-\frac{\alpha K_{0}(2 \phi)^{3 / 2}}{q^{3 / 2}}
$$

where $q$ is the coordination number of the lattice. The first term in Eq.(22) describes the self-avoidance of the chains. The second and the third term are the densities of ends and junctions, respectively, as can be seen from Eqs.(16). Denoting the volume fraction of ends by $\phi_{e}$ and of junctions by $\phi_{j}$ it follows:

$$
\begin{aligned}
\frac{h_{0}(2 \phi)^{1 / 2}}{q^{1 / 2}} & =\phi_{e} \\
\frac{\alpha K_{0}(2 \phi)^{3 / 2}}{q^{3 / 2}} & =\phi_{j}
\end{aligned}
$$

The expansion in powers of $h_{0}$ and $K_{0}$ is justified because we are interested in the limit where the density of ends is much smaller than the total monomer density, $\phi_{e} \ll \phi$. The density of junctions is always smaller than the total monomer density, $\phi_{j} \ll \phi$ as follows form Eq.(23) due to the fact that $K_{0}=e^{-\epsilon_{j} / T}<1$ for $\epsilon_{j}>0$.

We note in passing that the choice of the lattice affects only the numerical prefactors in Eqs.(23),(22). The lattice-specific dependence of junctions and ends densities in Eq.(23) on $q$ is consistent with a simple probabilistic argument. In equilibrium the probabilities of defect (e.g., ends and junctions) formation and break-up must be equal. The probability of bond formation is proportional to $\frac{1}{2} \phi_{e}^{2} q$, because a bond can be formed whenever two ends are 
neighbors on a lattice (the factor $\frac{1}{2}$ is due to indistinguishability of any two ends). The probability of bond break-up is proportional to the total number of bonds $N_{b} \approx N$. Taking into account that the formation of two ends from a single bond costs an energy $2 \epsilon_{e}$, the probability of a bond break-up is $\phi$ $e^{-2 \epsilon_{e} / T}$. Equating the probabilities of bond breaking and bond formation produces Eq. 23) (recalling that $h_{0}=e^{-\epsilon_{e} / T}$ ). Similarly, three ends can coalesce to form a junction, giving in equilibrium $\phi_{e}^{3} / e^{-3 \epsilon_{e}} \simeq \alpha \phi_{j} / e^{-\epsilon_{j}}\left(K_{0}=e^{\epsilon_{j} / T}\right)$. Note that this argument is independent of the fact whether the 'ground state' consists of infinite chains or closed rings of linear chains.

Together with Eq.(23), the free energy $f$ of Eq.(22) can be cast into the lattice-independent form

$$
f / T=\phi+(1-\phi) \ln (1-\phi)-\phi_{e}-\phi_{j}
$$

The first term in Eq. (24) is due to self-avoidance between the chains. This particular form of $f$ is expected on very general grounds. If one thinks of the ends and junctions as defects in the system of infinite chains, each defect lowers the free energy by $k_{B} T$, which is true for any system with non-conserved defects, e.g. dislocations in crystal structures.

\subsubsection{Average distance between the defects}

The average volume fractions of junctions, $\phi_{j}$, and ends, $\phi_{e}$, are not fixed but depend on the total volume fraction of monomers, $\phi$. Similarly, the self-assembling nature of the system means that the size distribution of the branched aggregates is polydisperse.

Absorbing the lattice-dependent prefactors in Eq.(23) into the definition of the constants $K$ and $h$, it is rewritten as:

$$
\phi_{j}=K_{0}^{\prime} \phi^{3 / 2} \text { and } \phi_{e}=h_{0}^{\prime} \phi^{1 / 2}
$$

As can be seen from Eq. 25) for $\phi \ll h_{0}^{\prime} / K_{0}^{\prime}$, the number of junctions is much smaller than the number of ends, $\phi_{j} \ll \phi_{e}$ while in the opposite limit $\phi_{j} \gg \phi_{e}$. Let us consider the mean length of a chain segment in between two consecutive defects (i.e., ends or junctions), $\bar{l}$. Each junction is attached to three chain segments while each end is attached to a single segment. The total length of the segments is proportional to the total volume of monomers in the system. Consequently $\left(\frac{3}{2} N_{j}+\frac{1}{2} N_{e}\right) \bar{l}=N$; the factor $\frac{1}{2}$ corrects for the 
double counting of each segment. Consequently, we find:

$$
\bar{l}=\frac{2 \phi}{3 \phi_{j}+\phi_{e}} \simeq \frac{2 \phi}{3 K_{0}^{\prime}(2 \phi)^{3 / 2}+h_{0}^{\prime}(2 \phi)^{1 / 2}}
$$

For $\phi \ll h_{0}^{\prime} / K_{0}^{\prime}$, the ends dominate and the mean chain length in between end points is $\bar{l} \sim \phi^{1 / 2}$ while for $\phi \gg h_{0}^{\prime} / K_{0}^{\prime}$ the junctions dominate and the mean chain length in between junction points is $\bar{l} \sim \phi^{-1 / 2}$. These results are in agreement with the known results for both non-branched micelles and pure networks [6, 41]. These results suggest, that at high densities (where the ends are negligible), a connected network is formed, as we shall actually prove in Sec. 5.2. They also imply that the mean chain length between defects, $\bar{l}(\phi)$ has a maximum around $\phi \sim h_{0}^{\prime} / K_{0}^{\prime}$. One can easily convince oneself taking the derivative $\frac{\partial \bar{l}}{\partial \phi}$ that $\bar{l}$ is indeed a non-monotonic function of $\phi$ whose maximum is located at $\phi=\frac{1}{2} \frac{h_{0}^{\prime}}{3 K_{0}^{\prime}}$ or, in other words, where $\phi_{e}=3 \phi_{j}$. This fact will become important for the determination of the details of the evolution of the system from a state of disconnected chains to that of a connected network (cf. Sec. 5.2).

In the absence of junctions (e.g. when the junction energy is very high and the resulting value of $K$ is close to zero), the mean chain length in between end points is $\bar{N}=\frac{\phi}{2 \phi_{e}}=h_{0}^{\prime} \phi^{1 / 2}$. The free energy $F\left(\phi, h_{0}\right)$ of Eq.(24) can be transformed to a free energy that depends only on $\phi$ and $\phi_{e}$ (or the mean chain length $\bar{N}$ ) by means of the Legendre transformation $f\left(\phi, h_{0}\right)+\phi_{e} \ln h_{0}=$ $\bar{f}(\phi, \bar{N})$. The result is identical to the Flory-Huggins expression for the free energy of the polymer solutions:

$$
\bar{f}(\phi, \bar{N})=(1-\phi) \ln (1-\phi)+\frac{\phi}{\bar{N}} \ln \phi
$$

As mentioned above, this is because the mean field approximation is geometrically equivalent to Flory lattice construction, which disregards the intrachain self-avoidance, but retains the excluded volume interactions between different chains. Thus our model describes the case of polydisperse chains with both ends and junctions as well as the limiting case of finite chains (with no junction points) where the average length is well defined; this is the case that is applicable to chemically prepared polymeric chains.

\subsection{The junctions-ends transition}


The free energy $f$ of Eq.(22) is unstable in a certain range of parameters ( monomer density $\phi$, defect energies $\epsilon_{e}$ and $\epsilon_{j}$ and temperature) and shows a first-order phase transition, terminating at a critical point. The spinodal line associated with this transition is determined by the condition $\frac{\partial^{2} F}{\partial \phi^{2}}=0$ and at the critical point, $\frac{\partial^{3} F}{\partial \phi^{3}}=0$. We determine the conditions for the critical point from our model as:

$$
\begin{aligned}
& \frac{\partial^{2} F}{\partial \phi^{2}}=\frac{1}{1-\phi}+\frac{h_{0}}{q^{1 / 2}}(2 \phi)^{-3 / 2}-3 \alpha \frac{K_{0}}{q^{3 / 2}}(2 \phi)^{-1 / 2}=0 \\
& \frac{\partial^{3} F}{\partial \phi^{3}}=\frac{1}{(1-\phi)^{2}}-3 \frac{h_{0}}{q^{1 / 2}}(2 \phi)^{-3 / 2}+3 \alpha \frac{K_{0}}{q^{3 / 2}}(2 \phi)^{-3 / 2}=0
\end{aligned}
$$

Recalling that $h_{0}=e^{-\epsilon_{e} / T}$ and $K_{0}=e^{-\epsilon_{j} / T}$, the Eq.(27) can be solved analytically for small $\phi$, expanding $(1-\phi)(\ln (1-\phi)-1) \simeq \frac{1}{2} \phi^{2}$, which gives

$$
\begin{aligned}
T_{c} & =\frac{-3 \epsilon_{j}+\epsilon_{e}}{\ln q^{4} /\left(4 \alpha^{3}\right)} \\
\phi_{c} & =\frac{1}{2} \frac{q}{\alpha} e^{\left(\epsilon_{j}-\epsilon_{e}\right) / T_{c}}
\end{aligned}
$$

while for arbitrary $\phi$ they can be solved numerically (Fig.5). It is interesting to note that in the small density limit, $\phi_{e} \approx \phi_{j}$ at the critical point, which emphasizes the fact that the transition is junctions-induced. For $T>T_{c}$ the system is a homogeneous mixture of chains and branched aggregates, while for $T<T_{c}$ there is a two-phase equilibria between an end-rich phase that coexists with a junction-rich phase. As will be shown in Sec. 5.2, the end-rich phase usually consists of dilute, disconnected chains, while the junction-rich phase is usually a connected network that spans the whole system volume.

Although there are no direct interactions between the monomers in our model, junction formation induces effective attraction between the monomers, and it is this effective interaction that leads to the phase separation of Eq.(27) . The Eq.(28) has several interesting consequences: First, the phase separation is possible only when $\epsilon_{j}<\frac{1}{3} \epsilon_{e}$ (as long as $q^{4} /\left(4 \alpha^{3}\right)>1$ ); in the opposite case, the junctions are present only as a minor 'perturbation' to a system of linear chains and their number is too small to generate an attraction large enough to drive a macroscopic phase separation. Second, the critical monomer density $\phi_{c}<\frac{1}{2}$; this fact is important for the understanding of the correlations between monomers and between junctions in the system. 
Third, if $\alpha$ is large $\left(q^{4} /\left(4 \alpha^{3}\right)<1\right)$, there is no upper critical temperature, and the phase separation takes place even at infinitely high temperatures $\left(T_{c} \rightarrow \infty\right)$. If the 'microscopic' end cap and junction energies, $\epsilon_{e}$ and $\epsilon_{j}$ themselves depend on temperature or density (e.g., in microemulsions [42]), the phase diagram may become more complicated. Note that the phase transition described by Eq.(27) shows reentrant behavior. At high temperatures, there is no phase separation, as usual. At very low temperatures the parameter $K_{0}=e^{-\epsilon_{j} / T}$ tends to zero and there is no separation either, as shown in Fig. 1, because the number of thermally generated junctions is too small to drive a phase separation.

The phase transition discussed here is of purely entropic origin, which augments a qualitative claim by De Gennes that the cross-links are thermodynamically equivalent to attractive interactions [1]. The origin of this phase transition lies in the fact that although the translational entropy of the chains is lower in the dense phase, the total entropy is still higher due to high entropy of the self-assembled junctions, which are abundant in the dense phase. It is important to emphasize that the presence of junctions induces an attraction between all monomers and not only between the junctions, as we shall show in Sec. . Also, the phase separation discussed here is not the same as the percolation (connectivity) transition, where an infinite cluster appears, although at both transitions a macroscopic connected network is formed. As we shall see in Sec. 5.2, the percolation transition, at which an infinite branched cluster appears in the system, is not a thermodynamic, but rather a structural transition. In some cases, the percolation transition can be masked by the end-junction phase separation. It is also interesting to note that for the values of $\epsilon_{e}$ and $\epsilon_{j}$ at which the phase separation transition is absent $\left(h_{0}>K_{0}\right)$, there is also no maximum in $\bar{l}(\phi)$, as follows from Eq.(26).

\subsubsection{Osmotic pressure and the phase coexistence line}

The osmotic pressure $\Pi$ can be calculated from the free energy $f$ of Eq.(22):

$$
\Pi=-f+\frac{\partial f}{\partial \phi} \phi \simeq-\ln (1-\phi)-\phi+\frac{1}{2} h_{0}^{\prime}(2 \phi)^{1 / 2}-\frac{1}{2} K_{0}^{\prime}(2 \phi)^{3 / 2}
$$

Note that the coefficient before $h_{0}^{\prime}$-dependent (ends) term is positive due to the fact that the exponent in $\phi^{1 / 2}$ is less than one, while the sign of the junctions term is negative. This emphasizes that fact that, although 
both ends and junctions terms are negative in the free energy, the junctions term is thermodynamically equivalent to an attraction, while the ends term produces an effective repulsion. The location of the phase coexistence line is determined as usual requiring that the chemical potentials $\mu=\frac{\partial f}{\partial \phi}$ and the osmotic pressures $\Pi$ in both phases are equal:

$$
\mu\left(\phi_{1}\right)=\mu\left(\phi_{2}\right) \quad \Pi\left(\phi_{1}\right)=\Pi\left(\phi_{2}\right)
$$

Numerical solution of these equations is shown in Fig. 11.

\subsection{Four-fold and higher functionality junctions: gela- tion vs. vulcanization}

The analysis of the previous section can be generalized to systems with crosslinks of arbitrary functionality $z$, by the addition to the Hamiltonian $\mathrm{H}_{3}$ of Eq.(10) a term that couples $z$ spins.

$$
-\sum_{i_{z}} K_{z} \underbrace{S_{1, i_{1}} S_{1, i_{2}} . . S_{1, i_{z}}}_{z \text { times }}
$$

with $K_{z}=J^{z / 2} e^{-\epsilon_{z}}$ (cf. Sec. ; $\epsilon_{z}$ being the energy of the $z$-fold junction. In this case the resulting Helmholtz free energy per unit volume then takes the form

$$
f / T \simeq(1-\phi)(\ln (1-\phi)-1)-\alpha_{1} h_{0} \phi^{1 / 2}-\alpha_{z} e^{-\epsilon_{z}} \phi^{z / 2}
$$

where $\alpha_{1}$ and $\alpha_{z}$ are two numerical, lattice dependent prefactors reflecting the local ends and junctions configurations, respectively. There are two interesting points to be noted.

(i) There is no thermodynamic phase separation for $z>4$, as can be readily seen from Eqs.(27, 29), because the resulting free energy of Eq. (29)

is always convex $\left(\frac{\partial^{2} f}{\partial \phi^{2}}>0\right)$. The attraction induced by the junctions weakens with increasing $z$. The topological percolation transition is, however, still present, as discussed in Sec. 5.2

(ii) When $z=4$, the four-fold junctions contribution to the free energy is indistinguishable from the two-body attractions between the monomers (at least at the mean-field level), both contributing term $\sim \phi^{2}$ to the free energy for $\phi \ll 1$. The free energy is

$$
f / T \simeq\left(\frac{1}{2}-\frac{1}{2} e^{-\epsilon_{4}^{\prime}}\right) \phi^{2}-\phi_{e}
$$


where the effective junction energy $\epsilon_{4}^{\prime}$ has been redefined to absorb the internal degrees of freedom of a junction, expressed in the prefactor $\alpha$ of Eq.(29). The term $\frac{1}{2} e^{-\epsilon_{4}^{\prime}}$ corresponds to the $\chi$ parameter of the Flory theory. At the point where $\epsilon_{4}^{\prime}=0$, the excluded volume repulsion is balanced by the junction-induced attraction, a situation analogous to the $\Theta$-point of polymer solutions. The location of the compensation point is intuitively very clear: if $\epsilon_{4}^{\prime}=0$, there is no cost for creating a junction and the chains can intersect each other at will (the fact that the junctions are four-fold is crucial!), thereby acting as completely Gaussian phantom chains.

The three- and four-fold junctions correspond to two physically distinct situations. Three- fold junctions describe the process of gelation when the monomers simultaneously cross-link and polymerize in the reaction bath. The clusters formed this way exhibit mostly three-fold junctions, simply due to the fact that the collision of three monomers is more probable that the four-fold collision. In addition, in some systems (e.g. microemulsions [42]) it can be shown that the energy of four-fold junctions is higher than that of three-fold junctions. The four-fold cross-links correspond to the process of vulcanization, when existing long polymer chains are cross-linked by addition

of cross-link molecules, by irradiation or other means [2, 4, 23]. Formation of three fold junctions is structurally inhibited by the nature of the cross-link which cross-links two preexisting chains. Contrary to gelation, these systems show only four-fold junctions that result from an intersecting pair of chains.

\section{Spatial variations and correlations.}

The advantage of the ' $n=0$ ' model is that it can be used to predict the spatial correlations of the monomers, ends and junctions, in addition to the thermodynamic properties discussed above. These correlations are obtained from calculations of the second moment of the relevant probability distributions, such as the two-point correlations of the physical parameters, e.g., the density. The density-density correlation function can be measured in scattering experiments. Apart from being interesting per se, the correlations can also provide information about structural, non-thermodynamic transitions which one might expect in such structurally complex systems. Here, we generalize the mean field treatment of the previous section to include the possibility of spatial variations; this formalism allows us to calculate the correlations in the system. Each spin is approximated by its ensemble average, 
Figure 3: Schematic illustration of the formation of a connected network form disconnected clusters upon increase in the monomer density $\phi$.

but a potential spatial variation in the value of the average spin (due for example, to a spatially varying external field) is allowed for (cf. Refs. [6, 47]). In this case one must allow for spatial variation of the constants, which are denoted now as $J_{i}, h_{i}, K_{i}$ each one labelled by its local spatial index. Neglecting the fluctuations of each spin about its local average value $\left\langle S_{i}\right\rangle$, as in Sec. 3, we have

$S_{i} S_{j}=\underbrace{\left(S_{i}-\left\langle S_{i}\right\rangle\right)\left(S_{j}-\left\langle S_{j}\right\rangle\right)}_{\text {neglected }}-\left\langle S_{i}\right\rangle\left\langle S_{j}\right\rangle+\left\langle S_{j}\right\rangle S_{i}+\left\langle S_{i}\right\rangle S_{j} \simeq-\left\langle S_{i}\right\rangle\left\langle S_{j}\right\rangle+\left\langle S_{j}\right\rangle S_{i}+\left\langle S_{i}\right\rangle S_{j}$

where $\left\langle S_{i}\right\rangle$ is a local spin average. Similarly we can approximate the triplet term as:

$$
\left.S_{i} S_{j} S_{k} \simeq-2\left\langle S_{i}\right\rangle\left\langle S_{j}\right\rangle\left\langle S_{k}\right\rangle+\left\langle S_{k}\right\rangle\left\langle S_{j}\right\rangle S_{i}+\left\langle S_{i}\right\rangle\left\langle S_{k}\right\rangle S_{j}+\left\langle S_{i}\right\rangle\left\langle S_{j}\right\rangle S_{k}\right)
$$

Using the same procedure as in Sec. 3 and writing the average of the local spin as $\left\langle S_{i}\right\rangle \equiv s_{i}$, we find that the grand-canonical potential per unit volume, 
$\omega\left\{s_{i}\right\}$ (cf. Appendix 8.4 for details) is:

$\omega\left\{s_{i}\right\} V=\frac{1}{2} \sum_{i j} J_{i} s_{i} s_{j}+2 \sum_{i j k} \frac{1}{3} K_{i} s_{i} s_{j} s_{k}-\sum_{i} \ln \left(1+\frac{1}{2}(\underbrace{\left.h_{i}+\sum_{n n_{i}} J_{i} s_{j}+3 \sum_{j, k} \frac{1}{3} K_{i} s_{j} s_{k}\right)^{2}}_{x_{i}})\right.$

where $\sum_{j k}^{(K)}$ is an unconstrained sum over all possible pairs of sites $j, p$, belonging to the same triple $i, j, k$. (cf. Appendix 8.2).

$$
\begin{aligned}
s_{i} & =-\frac{\partial \omega}{\partial h_{i}}=\frac{x_{i}}{1+\frac{1}{2} x_{i}^{2}} \\
\phi_{i} & =-\frac{\partial \omega}{\partial \ln J_{i}}=\frac{1}{2} \sum_{i j} J_{i} s_{i} s_{j}+\frac{1}{2} h_{i} s_{i}+\frac{3}{2} \sum_{i j k} K_{i} s_{i} s_{j} s_{k}=\frac{1}{2} s_{i} x_{i}
\end{aligned}
$$

From this we find:

$$
\begin{aligned}
x_{i} & =\sqrt{\frac{2 \phi_{i}}{1-\phi_{i}}} \text { and } s_{i}=\sqrt{2 \phi_{i}\left(1-\phi_{i}\right)} \\
J_{i} & =\frac{\left(x_{i}-h_{i}-K_{i} \sum_{j k}^{(K)} s_{j} s_{k}\right)}{\sum_{j} s_{j}}
\end{aligned}
$$

where $\sum_{j} s_{j}$ are sums over nearest neighbors of the site $i$. From these formulae, the Helmholtz free energy $F\left\{\phi_{i}\right\}=\omega V\left\{\phi_{i}\right\}+\sum_{i} \phi_{i} \ln J_{i}$ can be calculated.

For $K_{0}=h_{0}=0$ (corresponding to infinitely long chains) the free energy is

$$
F\left\{\phi_{i}\right\}=\sum_{i}\left[\phi_{i}+\ln \left(1-\phi_{i}\right)+\frac{1}{2} \phi_{i} \ln \frac{2 \phi_{i}}{1-\phi_{i}}-\phi_{i} \ln \sum_{n n_{i}}\left(2 \phi_{j}\left(1-\phi_{j}\right)\right)^{1 / 2}\right]
$$

which we explicitly write here to emphasize the very non-trivial coupling between the different $\phi_{i}$ 's coming from the underlined term. For a spatially uniform system, $\phi_{i}=\phi$, and the $F\left\{\phi_{i}\right\}$ has only the single term $(1-\phi) \ln (1-$ 
$\phi)$ of Eq. (22) because the terms proportional to $1 / \bar{N}$ vanish in the limit of $K_{0}=h_{0}=0$ (infinitely long chains) considered here for simplicity.

The focus of the present discussion is different from the treatment of Ref. [23], which was restricted to a study of spin-spin correlations in the context of the percolation transition. In this section, we focus on the physically measurable density-density correlations and the thermodynamic properties. The location of the percolation line can also obtained within slightly modified formalism, at least at the mean field level, allowing to relate the thermodynamic properties of the system to its structure.

\subsection{Density fluctuations}

Extending the mean-field theory to treat small, local density fluctuations, the free energy as a function of the local densities, $F\left\{\phi_{i}\right\}$ is systematically expanded to quadratic order in the density fluctuations $\delta \phi_{i}$, related to the local average value of the density and the mean density by the relation: $\phi_{i}=\phi+\delta \phi_{i}$.

$$
F\left\{\phi_{i}\right\}=F_{M F}(\phi)+\sum_{i} \underbrace{\ln J_{i}}_{\frac{\partial F}{\partial \phi_{i}}} \delta \phi_{i}+\underbrace{\sum_{k i} S_{k i}^{-1} \delta \phi_{k} \delta \phi_{i}}_{\delta^{(2)} F}+O\left(\delta \phi^{3}\right)
$$

where $F_{\mathrm{MF}}(\phi)$ is just the mean field free energy of Sec. 3; $F_{\mathrm{MF}}(\phi)=V f_{\mathrm{MF}}(\phi)$, $S_{i k}^{-1}=\frac{\partial^{2} F\left\{\phi_{i}\right\}}{\partial \phi_{i} \partial \phi_{k}}$ is a matrix of the second derivatives of $F\left\{\phi_{i}\right\}$, and we have used the fact that $\frac{\partial F}{\partial \phi_{i}}=\mu_{i}=\ln J_{i}$. Expansion in Eq.(35) is a completely general one, and the particular expressions for $S_{i k}^{-1}$ can be obtained from Eqs. (33,32). By the definition of the constant $J_{i}$, as an exponent of a local chemical potential, the density-density fluctuation function is given by $\left\langle\phi_{i} \phi_{k}\right\rangle-\phi^{2}=\frac{\partial^{2} \ln Z\left\{\phi_{i}\right\}}{\partial \ln J_{i} \partial \ln J_{k}}$ which turns out to be the inverse of the matrix $S_{i k}^{-1}$ :

$$
\left\langle\phi_{i} \phi_{k}\right\rangle-\phi^{2}=\frac{1}{V} \frac{\partial \phi_{i}}{\partial \ln J_{k}}=\frac{1}{V} S_{i k}
$$

where we have used the fact that $\frac{\partial \ln Z\left\{\phi_{i}\right\}}{\partial \ln J_{i}}=\phi_{i}$. A cumbersome but straightforward calculation using the Eq.(33)(cf. Appendix 8.3 ) gives 


$$
\begin{aligned}
\frac{\partial^{2} F\left\{\phi_{i}\right\}}{\partial \phi_{i} \partial \phi_{k}}= & \frac{\partial \ln J_{i}}{\partial \phi_{k}}=\frac{1}{2 \phi(1-\phi)}\left(\delta_{i k}-(1-2 \phi) \frac{1}{q} \sum_{j=n n_{i}} \delta_{j k}\right)+ \\
& +\frac{h_{0}}{2 q^{1 / 2}(2 \phi)^{3 / 2}} \frac{1}{(1-\phi)}\left(\delta_{i k}+(1-2 \phi) \frac{1}{q} \sum_{j=n n_{i}} \delta_{j k}\right) \\
& -\frac{3 K_{0}\left(\alpha^{\prime} / 3\right)}{q^{3 / 2}(2 \phi)^{1 / 2}} \frac{1}{(1-\phi)}\left(\frac{1}{2} \delta_{i k}-(1-2 \phi) \frac{1}{6 q} \sum_{j=n n_{i}} \delta_{j k}+\frac{2}{\alpha^{\prime}}(1-2 \phi) \sum_{j=n n n_{i}} \delta_{j k}\right)
\end{aligned}
$$

Writing the Fourier transform

$$
\delta \phi_{i}=\sum_{\vec{p}} \delta \phi(\vec{p}) e^{i \vec{p} \cdot \vec{r}_{i}}
$$

we get

$$
\delta^{(2)} F=\sum_{\vec{p}} S^{-1}(\vec{p}) \delta \phi(\vec{p}) \delta \phi(-\vec{p}) \text { and }\langle\delta \phi(\vec{p}) \delta \phi(-\vec{p})\rangle=\frac{1}{V} S(\vec{p})
$$

with $S(\vec{p})=\sum_{i k} S_{i k} e^{i \vec{p}\left(\vec{r}_{i}-\vec{r}_{k}\right)}$. From Eq.(36) we find (for the simple cubic lattice)

$$
\begin{aligned}
S^{-1}(\vec{p})= & \frac{1}{2 \phi(1-\phi)}\left[A+B\left(\cos \left(p_{x}\right)+\cos \left(p_{y}\right)+\cos \left(p_{z}\right)\right)+\right. \\
& \left.+C\left(\cos \left(p_{x}\right) \cos \left(p_{y}\right)+\cos \left(p_{z}\right) \cos \left(p_{y}\right)+\cos \left(p_{x}\right) \cos \left(p_{z}\right)\right)\right]
\end{aligned}
$$

where

$$
\begin{aligned}
A & =1+\frac{h_{0}}{2(2 \phi q)^{1 / 2}}-\frac{3 K_{0}\left(\alpha^{\prime} / 3\right)}{2 q^{3 / 2}}(2 \phi)^{1 / 2} \\
B & =(2 \phi-1)\left[\frac{2}{q}-\frac{h_{0}}{(2 \phi)^{1 / 2} q^{3 / 2}}-\frac{3 K_{0}(\alpha \prime / 3)}{3 q^{5 / 2}}(2 \phi)^{1 / 2}\right] \\
C & =(2 \phi-1) \frac{4 K_{0}(\alpha \prime / 3)}{q^{5 / 2}}(2 \phi)^{1 / 2}
\end{aligned}
$$

and we have used the fact that the Fourier transform $(F T)$ of the delta function is equal to unity and

$$
F T\left[\sum_{j} \delta_{i j}\right]=\sum_{\alpha} e^{i p_{\alpha} \hat{e}_{\alpha}}
$$


where $\hat{e}_{\alpha}$ is the unit vector that points in the direction of the site $j$. On a simple cubic lattice, $p_{\alpha}$ is the component of the vector $\vec{p}$ in the direction $\alpha$. As one can easily see from Eqs.(22, 37), the inverse of the structure factor at the zero wavevector $S(0)=\int S(r) d r$ is equal to the second derivative of the mean field free energy:

$$
S^{-1}(0)=\frac{\partial^{2} F_{\mathrm{MF}}(\phi)}{\partial \phi^{2}}=\frac{1}{1-\phi}+\frac{h_{0}}{(2 \phi)^{3 / 2} q^{1 / 2}}-\frac{3 K_{0}(\alpha \prime / 3)}{(2 \phi)^{1 / 2} q^{3 / 2}}
$$

This is an expression of the fluctuation-dissipation theorem in the grandcanonical ensemble. The variance of the mean number of monomers in the grand canonical ensemble is

$$
\left\langle N^{2}\right\rangle-\langle N\rangle^{2}=\int d r\left\langle(\phi(r)-\phi(0))^{2}\right\rangle=T V \phi^{3} \kappa_{T}=S(0)
$$

where $\kappa_{T}$ is the isothermal compressibility 8$]$. Noting that

$$
\frac{\partial^{2} F_{\mathrm{MF}}(\phi)}{\partial \phi^{2}}=\frac{\partial \mu}{\partial \phi}=\frac{1}{\phi^{3}} \frac{\partial \Pi}{\partial v}=\frac{1}{\phi^{3}} \kappa_{T}^{-1}
$$

( $\Pi$ is the osmotic pressure, $v=1 / \phi$ and $\mu=\partial F_{\mathrm{MF}}(\phi) / \partial \phi$ is the chemical potential), Eq.(38) becomes identical to Eq.(39). Thus, the calculated density-density correlation function satisfies the expected thermodynamic sum rules.

The structure factor $S(\vec{p})=\langle\delta \phi(\vec{p}) \delta \phi(-\vec{p})\rangle$ is experimentally measurable by scattering experiments (neutron, light, X-ray etc.) where the intensity of scattered radiation at the wavevector $\vec{p}$ is proportional to $S(\vec{p})$. If $S(0)$ is determined experimentally as a function of $\phi$, the free energy $F_{\mathrm{MF}}(\phi)$ can be found by the integration of Eq.(38). Obviously, Eq.(37) is not valid for the values of parameters at which the phase separation is observed, that is, in the region where $\frac{\partial^{2} F(\phi)}{\partial \phi^{2}}<0$. Rather, each macroscopic phase in a two-phase equilibrium has its own value of $S(\vec{p})$, with the value of the monomer density appropriate for each phase.

The expression (37) for $S(\vec{p})$ is quite complicated, and its behavior is rather different for low and high densities. We next consider the nature of the predicted structure factor of Eq.(37) in the limits of high and low monomer density, $\phi$. 


\subsubsection{Low density limit}

For small densities $\phi$ the coefficients $B$ and $C$ are negative, and one can expand Eq.(37) in a power series in the wavevector $\vec{p}$. One finds that the coefficient of $p^{2}$ is positive so that this expansion is reasonable for wavevectors that are small compared with the inverse of the monomer size.

$$
\begin{aligned}
S^{-1}(\vec{p}) & \simeq S^{-1}(0)+a p^{2} \text { so that } S(\vec{p}) \simeq \frac{S(0)}{1+a S(0) p^{2}} \\
S(0) & =\left(\frac{\partial^{2} F_{\mathrm{MF}}(\phi)}{\partial \phi^{2}}\right)^{-1} \text { and } a=\frac{(1-2 \phi)}{2 \phi(1-\phi)}\left(1+\frac{3 K_{0}\left(\alpha^{\prime} / 3\right)}{q^{3 / 2}} \frac{15}{4}(2 \phi)^{1 / 2}-\frac{h_{0}}{(2 \phi q)^{1 / 2}}\right)
\end{aligned}
$$

Note that $a$ is a non-negative, because $\frac{h_{0}}{(2 \phi q)^{1 / 2}}=\frac{\phi_{e}}{2 \phi}$, the half of the ratio of the number of ends to the number of junctions. In the present approximation of sparse junctions and ends, $\frac{\phi_{e}}{\phi} \ll 1$ and $a$ is non-negative. In the case of linear polymers without junctions, $\frac{\phi_{e}}{2 \phi}$ is equal to the inverse average length of the chains, $\frac{\phi_{e}}{2 \phi}=\frac{1}{N}$, which leads to the known correction to the scattering structure factor [2].

In real space, the density-density correlation function is given by Fourier transform of the $S(\vec{p})$. It follows from Eq. (40) that in real space $\langle\delta \phi(\vec{r}) \delta \phi(0)\rangle$ has the usual Ornstein-Zernicke form

$$
\langle\delta \phi(\vec{r}) \delta \phi(0)\rangle=\sum_{\vec{p}} S(\vec{p}) e^{i \vec{p} \cdot \vec{r}} \simeq \frac{1}{r} e^{-r / \xi}
$$

with the correlation length $\xi$ given by

$$
\xi=a\left(\frac{\partial^{2} F_{\mathrm{MF}}(\phi)}{\partial \phi^{2}}\right)^{-1}
$$

The correlation length of density fluctuations, $\xi$, diverges at the spinodal line of the first-order junction-ends transition studied in the Sec.3.2, at which $\frac{\partial^{2} F_{\mathrm{MF}}(\phi)}{\partial \phi^{2}}=0$; this is expected for any first-order transition. Similarly, from Eq.(40) we see that the density fluctuations at zero wavevector, $S(\vec{p}=0)$, are also divergent at the spinodal, and, in particular, at the critical point. The divergence of the scattering intensity at the critical point should be observable in scattering experiments in the same way as the usual critical opalescence. We note in passing that in the absence of junctions, the structure factor, as 
given by Eq. (40), reduces to the classical RPA result for polymer solutions [2].

It is interesting to note that the overall structure of a solution of selfassembled branched aggregates is not strictly self-similar, as we can see from Eq. (40) . There is at least one characteristic length in the problem, namely, the correlation length $\xi$. At distances larger than $\xi$ the correlations decay exponentially while for self-similar structures one expects algebraic decay of correlations. However, each branched cluster is expected to be self-similar, in analogy to percolation clusters 24. Note that both the correlation length $\xi$ and the scattering intensity at zero wavevector $S(0)$ are both proportional to the second derivative of the free energy, $\frac{\partial^{2} F_{\mathrm{MF}}(\phi)}{\partial \phi^{2}}$. This means that both of them are non-monotonic functions of the density, but have a maximum around the line $\phi_{e} \approx \phi_{j}$, as follows from Eqs.(27) (41), by taking the derivatives $\frac{\partial \xi}{\partial \phi}, \frac{\partial S(0)}{\partial \phi}$. The maxima of $S(0)$ and of $\xi$ as a function of $\phi$ are determined by the condition $\frac{\partial S(0)}{\partial \phi}=\frac{1}{S(0)^{2}} \frac{\partial^{3} F_{\mathrm{MF}}(\phi)}{\partial \phi^{3}}=0$, which is precisely the same condition which determines the location of the critical point $\left(\left.\frac{\partial^{3} F_{\mathrm{MF}}(\phi)}{\partial \phi^{3}}\right|_{\phi_{c}}=0\right)$. In particular, this leads to the conclusion that the maximum scattering in the region above the phase separation should be observed along a line which starts at the critical point and is determined by the condition $\frac{\partial^{3} F_{\mathrm{MF}}(\phi)}{\partial \phi^{3}}=0$ ( equivalent for the small densities to the condition $\phi_{e} \approx \phi_{j}$ ), as shown in Fig. 5. This is in agreement with observations from scattering experiments from solutions of branched wormlike micelles [33](cf. also Discussion).

It is instructive to compare $\xi$ with another length scale that is present in the problem, namely, $\bar{l}$, the mean distance between the defects (i.e. ends or junctions). As shown in Sec.3,

$$
\bar{l}=\frac{2 \phi}{3 \phi_{j}+\phi_{e}} \simeq \frac{\phi}{3 K_{0}^{\prime} \phi^{3 / 2}+h_{0}^{\prime} \phi^{1 / 2}}
$$

and $\bar{l}(\phi)$ has a maximum around $\phi \sim h_{0}^{\prime} / K_{0}^{\prime}$. The behavior of $\bar{l}$ is quite different from that of $\xi$, indicating that these two lengths are physically unrelated. The behavior of $\bar{l}$ is non-singular, while the correlation length $\xi$ diverges at the spinodal line of the junction-ends transition. This emphasizes the fact that the junction-induced attraction has an effect on all the monomers in the system, as reflected in the behavior of $\xi$; the effect of junction induced attraction is not limited to the behavior of the junctions alone; this is reflected in the non-singular nature of $\bar{l}$. The physical meaning of $\xi$ is similar to the 
'blob' size of the semidilute polymer solutions. Namely at distances smaller than $\xi$, the behavior of an aggregate is that of a single self-avoiding branched polymer.

\subsubsection{High densities}

When $\phi>1 / 2$, the coefficient $(2 \phi-1)$ in Eq.(37) becomes positive, which results in a negative coefficient in front of the term proportional to $p^{2}$ (in the expansion of the structure factor for small wavevectors). Thus, the small wavevector expansion becomes meaningless, since it would give a negative structure factor for high enough values of $p$. Therefore, in this region one cannot use the expansion and one must retain the full form of the $S(\vec{p})$. The exact location of the line where the behavior changes, $\phi=1 / 2$, is an artifact of the lattice model and should not be taken literally. Inspection of Eq.(37) reveals that for small enough junctions densities, ( small Boltzmann factor $\left.K_{0}=e^{-\epsilon_{j} / T}\right)$, for which

$$
|B /(2 C)|<1 \Rightarrow K_{0}^{\prime}<\frac{4}{17} \frac{\left(1-\frac{1}{2} \frac{h_{0}}{(2 \phi q)^{1 / 2}}\right)}{(2 \phi)^{1 / 2}} \equiv K^{\star}
$$

$\left(K_{0}^{\prime}=K_{0} \alpha / q^{3 / 2}\right)$, the structure factor $S(\vec{p})$ is a monotonically increasing function of the wavevector, $p$ (for concreteness we consider the $[1,1,1]$ direction) for all $\phi>1 / 2$.

This behavior of the structure factor at small junctions densities (weakly branched chains) agrees with the previous studies of the density fluctuations in concentrated polymer solutions and melts [30]. Indeed, it is known that the structure factor of concentrated polymer solutions and melts behaves qualitatively like that of simple liquids [30]. In particular, for small wavevectors $p$, the $S(p)$ is an increasing function of $p$. This is related to the low compressibility of dense liquids. As discussed in the previous section, as the monomer density $\phi$ tends to one, the compressibility tends to zero. The magnitude of the density fluctuations, related to the compressibility by the fluctuationdissipation theorem tends to zero as well [8]. When the monomer density $\phi$ is high, but lower than one, the long wavelength (small $p$ ) density fluctuations are small, because large collective rearrangements of the monomers are structurally inhibited. However, short wavelength, local, rearrangements of the molecules are still possible. Combined together, these two effects produce a structure factor that increases with increasing wavevector, $p$. 
In real systems, for the values of $p$ larger than the inverse molecular size, one observes oscillations of the structure factor as a function of $p$, with the peaks corresponding to the first-shell, second-shell, etc., correlations of the positions of the adjacent monomers. Although the lattice model in the mean field approximation cannot be used to predict precisely the extremely low short scale features, such as the first shell peak observed in scattering experiments on polymer solutions, it adequately treats the qualitative behavior of the structure factor for wavevectors smaller than the inverse of the lattice constant.

For higher values of $K_{0}, K^{\prime}>K^{\star}$, (i.e. for higher junction densities) a peak emerges in $S(\vec{p})$ ( see Fig. 5) at a value of wavevector given by:

$$
p_{0}=\arccos \left[-\frac{B}{2 C}\right]
$$

indicating the presence of medium range correlations. This peak is quite different form the first-shell peak of simple fluids, which reflects excluded volume interactions on the molecular scale. On the contrary, the peak of Eq.(43) reflects medium range structural correlations. By medium range we mean that the correlation range is larger than the lattice constant. The location of the peak depends on the values of the parameters, as discussed below, and it is not a lattice artifact. Because the peak appears only deep in the network phase, where the number of ends is negligible compared to the number of the junctions, one can neglect the $h_{0}$-dependent term in Eq.(37) as a first approximation and write:

$$
p_{0} \cong \arccos \left[\frac{1}{4}\left(1-\frac{1}{4 K_{0}^{\prime}(2 \phi)^{1 / 2}}\right)\right]
$$

indicating that the location of the peak moves towards lower values of $p$ as $K_{0}^{\prime}(2 \phi)^{1 / 2}$ increases. Fig. $\bigoplus$ shows the structure factor for two values of $K_{0}^{\prime}$ : below and above $K^{\star}$. Although Eq.(44) was obtained for the [1,1,1] direction $\left(p_{x}=p_{y}=p_{x}\right)$, a similar result would be obtained for the isotropically averaged structure factor $\bar{S}(p)=\int d^{3} p S(\vec{p})$. The peak in $\bar{S}(p)$ should appear whenever there is a peak in any 'crystallographic' direction. In isotropic systems, the averaged structure factor $\bar{S}(p)$ is, of course, the experimentally measurable quantity, free of lattice and model artifacts. The predicted peak in the structure factor due to the presence of the junctions, is not a lattice artefact, because the location of this peak location is not related to the lattice 
Figure 4: Structure factor as a function of wavevector $p$ in the high density region, $\phi>\frac{1}{2}$. Dashed line shows the structure factor at low tempertaures, where the number of thermally-generated junctions is small. For higher temperatures, a peak developes in the structure factor, shown by the thick line.

constant, but determined solely by the numbers of junctions present in the system.

The precise size of the junction is arbitrary to a certain extent and can be adjusted to reflect the real physical features of any given system. We have defined a junction as a connection between three adjacent sites. However, one can also consider a larger junction, connecting next nearest neighbors, as in Fig. 8(b). Intuitively, one expects a more pronounced peak for larger junctions, because it amounts to an increase of the junction volume. Exact numerical calculations show that it is indeed the case.

In the context of microemulsions, this peak can be related to the experimentally observed peak in the scattering from bicontinuous microemulsions [5, 43] (cf. Discussion). It is important to realize that the absence of a peak does not, in principle, imply the absence of a network, (cf. Fig. 5). 


\section{Spin-spin correlations and topological struc- ture.}

So far we have been concerned with thermodynamic properties of a system and related phenomena such as equilibrium density fluctuations. They can be adequately described by a model presented in Sec. 2.2 which maps the solution of equilibrium branched clusters onto Heisenberg model with anisotropic $S_{i, 1} S_{j, 1} S_{k, 1}$ three-spin term. However, no structural information, e.g., concerning the formation of a continuous network, can be extracted from the model as it is formulated in Sec. 2.2. Unfortunately, in order to be able to extract structural information about the network topology from the model, while retaining the exact correspondence between the spin model and the physical system of branched clusters, one has to resort to tensor order parameter, which results in a formally complicated theory 23. Instead, we shall use a simpler, but less rigorous theory, applicable only in the mean field approximation, employed in this paper.

Although the spin $\vec{S}$ has 'zero' components, one can still think about a 'parallel' component $S_{1}$ pointing in the direction of the field $\vec{h}$ and a 'transverse' component $S_{\perp}$. The rigorous mathematical procedure that justifies this is shown in Appendix. 8.4 and amounts to calculating all the quantities of interest for a finite number of components $n$, and taking the limit $n \rightarrow 0$ subsequently. One can then devise a slightly more generalized version of the Hamiltonian 10, where the three-spin term takes the following form:

$K \sum_{i j k}\left[S_{i, 1} S_{j, 1} S_{k, 1}+\frac{\beta}{3}\left(S_{i, 1}\left(S_{j, \perp} \cdot S_{k, \perp}\right)+S_{j, 1}\left(S_{i, \perp} \cdot S_{k, \perp}\right)+S_{k, 1}\left(S_{i, \perp} \cdot S_{j, \perp}\right)\right)\right]$

where the sum is over distinct triples $i j k$ and $\beta$ is a numerical parameter. One can also include coupling between the 'transverse' components of the spin $\vec{S}$, consistent with the symmetries of the system. This modification of the Hamiltonian has no effect on the thermodynamic properties and equilibrium fluctuations in the mean field approximation, discussed so far. Calculation of either the partition function or the two-point correlation function has contributions from the terms proportional to $\beta$ that have at least one power of the average of the transverse component $\left\langle S_{\perp}\right\rangle$. Since $\left\langle S_{\perp}\right\rangle=0$, terms proportional to $\beta$ do not contribute to thermodynamic quantities at the level of the mean field approximation. For $\beta=0$ the Hamiltonian (45) reduces 
to the previously used one. For $\beta \neq 0$ the correspondence between the spin model and the equilibrium branched clusters is not exact. The clusters containing internal loops enter into expansion with the wrong weight due to summation over $n-1$ 'transverse' components. However, with the choice $\beta=3$ all loopless tree-like clusters are counted correctly (cf. Appendix 8.4). Mean field approximation neglects the presence of intra-cluster loops, which formally correspond to correlations between different spins. This can be seen from the fact that the mean field free energy does not depend on the value of $\beta$ in Eq.(45). That is, it is independent of the precise way in which weights are assigned to the internal loops, which means that the intra cluster loops are neglected in the mean field approximation (cf. also Appendix 8.4). Thus, at the mean field level, the Eq.(45) with $\beta=3$ can be used to calculate the transverse spin correlations which are related to the cluster size and percolation threshold. Therefore, we use the $\beta=3$ model which neglects inter-cluster long-range correlations, responsible for the loops, consistently with the mean field approximation.

Although the spin $\vec{S}$ itself has no physical meaning, it enters the calculation of the various physical quantities, as we have seen in Sec. 3. Analogously, the spin-spin correlation functions $\left\langle S_{i, a} S_{j, a}\right\rangle$, although unphysical themselves, can be used to derive physically relevant quantities such as the density-density correlation function. Another interesting, physical observable (e.g., by scattering experiments conducted in solutions of end-labelled chains) is the correlation between the end-points of the aggregates, which is related to the spin-spin correlation function by (cf. Appendix. 8.4)

$$
\left\langle\phi_{e}\left(r_{i}\right) \phi_{e}\left(r_{j}\right)\right\rangle-\phi_{e}^{2}=\frac{\partial h_{i}\left\langle S_{i}\right\rangle}{\partial h_{j}}=\delta_{i j}+h\left[\left\langle S_{i} S_{j}\right\rangle-\left\langle S_{i}\right\rangle\left\langle S_{j}\right\rangle\right]
$$

This can be understood geometrically by noting that the graphs which enter into $\left\langle S_{i} S_{j}\right\rangle$ are those with ends present both at the site $i$ and site $j$. (cf. Fig. 6 and Appendix 8.4).

Similarly, although $S_{\perp}$ itself does not have a direct physical interpretation, its correlator $\left\langle S_{i, \perp} S_{k, \perp}\right\rangle$ does. If one considers the expansion of the correlation function $\left\langle S_{i, \perp} S_{k, \perp}\right\rangle$ (cf. Appendix 8.4) in powers of $J, h$ (in the absence of junctions, i.e., $K=0$ ) one can see that the only non-zero contributions arise from configurations that contain a single chain which starts at the site $i$ and ends at the site $k$. Consequently, $\left\langle S_{i, \perp} S_{k, \perp}\right\rangle$ measures the correlations between the ends of a single chain. As a matter of fact, even when $K \neq 0$, $\left\langle S_{i, \perp} S_{k, \perp}\right\rangle$ is non-zero only when $i$ and $k$ are the ends belonging to the same 
cluster (cf. Appendix 8.4 and Fig. 6). Thus, in general, the 'transverse' correlation function measures the correlations between chain ends belonging to the same aggregate. Similarly the 'longitudinal' correlation function $\left\langle S_{i, 1} S_{k, 1}\right\rangle$ measures the end-end correlations between any two ends, even if they belong to different chains or aggregates. The density-density correlations can be calculated from the 'longitudinal' correlator $\left\langle S_{i, 1} S_{k, 1}\right\rangle$ using Eq. (32) that relates $\phi_{i}$ to $S_{i}$. This calculation yields result identical to those obtained in Sec. 8.3.

In this section, we present the results of a calculation of the spin-spin correlators $\left\langle S_{i, a} S_{j, a}\right\rangle$ using the same local mean field approximation which was used in Sec. 3 to find the density-density correlation function. Each spin is approximated by its local ensemble average: $s_{i, \alpha}=\left\langle S_{i, \alpha}\right\rangle_{\text {local }}$ (with $a=\{1, \perp\}$ ), which can, however, be different for spins at different sites labelled by $i$. Eventually, we will be interested in the deviations $\delta s_{i}$ of the local average spins from the spatially averaged value $S$. The full calculation is presented in Appendix. 8.4. The spin-spin correlator is obtained from the partition function $Z$ using the relations:

$$
\left\langle\delta s_{i, a} \delta s_{j, a}\right\rangle=\frac{\partial^{2}}{\partial h_{i} \partial h_{j}} \ln Z=\frac{\partial s_{i, a}}{\partial h_{j, a}}
$$

Using the results of Eq.(32), a tedious but straightforward calculation yields the Fourier transform of the spin-spin correlator, $C_{\vec{p}}^{a}=\sum_{\vec{p}} e^{i \vec{p}\left(\vec{r}_{i}-\vec{r}_{k}\right)}\left\langle\delta s_{i, a} \delta s_{j, a}\right\rangle$ :

$$
C_{\vec{p}}^{a}=\frac{A^{a}}{1-A^{a}\left(J g_{1}(\vec{p})+6 K s g_{2}(\vec{p})\right)}
$$

where (cf. Appendix. 8.4)

$$
\begin{aligned}
A^{(1)} & =(1-2 \phi)(1-\phi) \\
A^{(\perp)} & =(1-\phi)
\end{aligned}
$$

and where

$$
\begin{aligned}
& g_{2}(\vec{p})=\frac{2}{3} \frac{\alpha}{q} \sum_{\text {unit cell }} e^{i \vec{p} \hat{e}_{\alpha}}+\sum_{\text {second cell }} e^{i \vec{p} \hat{e}_{\alpha}} \\
& g_{1}(\vec{p})=\sum_{\text {unit cell }} e^{i \vec{p} \hat{e}_{\alpha}}
\end{aligned}
$$


Figure 5: At low densities, $\phi<\frac{1}{2}$, the structure is a decreasing function of thewavevector. The scattering intensity at zero wavevector and the correlation length diverge at the phase stability coundary of the junctons-ends transition, shown by the thick closed line. In the region above the phase separation, the scattering intensity has a maximum along the dashed line, starting at the critical point. For high densities and low numbers of junctions, the structure factor is an increasing function of the wavevector, while for higher numbers of junctions present, a peak develops in the structure factor

Consequently, the spin-spin correlation function in Fourier space is:

$$
\left\langle\delta s_{a}(\vec{p}) \delta s_{a}\left(\vec{p}^{\prime}\right)\right\rangle=C_{\vec{p}}^{a} \delta_{\vec{p},-\vec{p}^{\prime}}
$$

In the following we show these results can be used to determine various physical characteristics of the system.

\subsection{Longitudinal correlations and the defect correla- tion function}

The correlation function of the aggregate ends can be calculated form Eq.(47), giving in Fourier space

$$
\begin{aligned}
\left\langle\delta \phi_{e}(\vec{p}) \delta \phi_{e}(-\vec{p})\right\rangle & =1+h C_{\vec{p}}^{1}= \\
\left\langle\delta \phi_{e}(\vec{p}) \delta \phi_{e}(-\vec{p})\right\rangle-1 & =h(1-2 \phi) S(\vec{p})
\end{aligned}
$$


where $S(\vec{p})$ is the same as in Eq. (37) for density-density correlations. Apart from the prefactor and the constant ( equal to 1) that ensures that the correlation of an end with itself is not counted, the dependence of $\left\langle\delta \phi_{e}(\vec{p}) \delta \phi_{e}(-\vec{p})\right\rangle$ on the wavevector $p$ is identical to that of the full density-density correlation function of Sec. 8.3. This is not accidental, but rather is a direct consequence of the fact that the density-density correlation function is related to spin-spin correlation function via Eq.(32). In particular, the correlation length at small densities $\xi$ is the same as the correlation length of the total density fluctuations. The same is true of the junction-junction correlation function

$$
\left\langle\phi_{j}\left(r_{i}\right) \phi_{j}\left(r_{j}\right)\right\rangle-\phi_{j}^{2}=\frac{\partial K_{i} \sum_{i, k, m} s_{i} s_{k} s_{m}}{\partial K_{j}}=\delta_{i j} K \alpha s^{3}+6 K s^{2} \sum_{p, j}^{(K)} \frac{\partial s_{p}}{\partial K_{j}}
$$

which can be calculated in a manner similar to that used to calculate $\left\langle S_{i} S_{j}\right\rangle$, and the subscript $(K)$ indicates that the summation is over all possible couples of the spins belonging to the original triple $i, k, m$.

The fact that the correlation length of total density fluctuations equals that of the ends/junctions fluctuations emphasizes the point that the junctions induce an overall effective attraction between the monomers and not only between the defects (i.e., ends and junctions). In particular, the correlation length of junction-junction correlations is the same as that of the monomer density-density correlations. The correlation length, related to the thermodynamics of the system, is unrelated to the mean distance $\bar{l}$ between the junctions which reflects the structural and topological properties.

\section{2 'Transverse' correlations: the structure of a single aggregate and percolation}

As have been mentioned, the 'transverse' correlation function measures the autocorrelations (denoted by the subscript auto) between the ends of the same cluster

$$
\left\langle\phi_{e}\left(r_{i}\right) \phi_{e}\left(r_{j}\right)\right\rangle_{\text {auto }}=h^{2}\left\langle S_{i}^{\perp} S_{j}^{\perp}\right\rangle
$$

Because $A^{\perp}=(1-\phi)$ is always positive, we can safely expand the expression for the spin-spin correlator of Eq.(47) up to second order in the wavevector $p$. Substituting the mean field values of $J$ and $K$ from Eq.(21), one gets 
Figure 6: 'Longitudinal' spin-spin correlator measures the correlations between any two chain ends. The 'transverse' correlator measures the correlation between the ends of the same chain.

$C_{\vec{p}}^{\perp}=\frac{(1-\phi)}{1-(1-\phi) A^{a}\left(J g_{1}(\vec{p})+6 K s g_{2}(\vec{p})\right)} \simeq \frac{1}{h_{0} /(2 \phi)^{1 / 2}-3 K_{0} \alpha(2 \phi)^{1 / 2}+b p^{2}}$

where $b$ is a non-singular function of the parameters. In other words

$$
\begin{aligned}
\left\langle\phi_{e}(\vec{p}) \phi_{e}\left(\vec{p}^{\prime}\right)\right\rangle_{\text {auto }} & =\frac{1}{\left(\phi_{e}-3 \phi_{j}\right) / 2 \phi+b p^{2}} \delta_{\vec{p},-\vec{p}^{\prime}} \\
\left\langle\phi_{e}(r) \phi_{e}(0)\right\rangle_{\text {auto }} & \simeq \frac{1}{r} e^{-r / L}
\end{aligned}
$$

with $L=\left(2 \phi /\left(\phi_{e}-3 \phi_{j}\right)\right)^{1 / 2}$. This means that the correlations between the ends belonging to the same aggregate are negligible for $r>L$. For large separations $r$, the dominant contribution to $\left\langle\phi_{e}(r) \phi_{e}(0)\right\rangle$ comes from the largest cluster. In other words, the average size of the largest cluster is $\bar{R} \simeq L$

$$
\bar{R} \simeq\left(\frac{2 \phi}{\left(\phi_{e}-3 \phi_{j}\right)}\right)^{1 / 2}
$$


When $\phi_{j}=\frac{1}{3} \phi_{e}$, the mean size of the largest diverges, and an infinite cluster is formed. This process is known as percolation and the emergence of an infinite network at $\phi_{j}=\frac{1}{3} \phi_{e}$ agrees with other mean-field level studies of percolation [23], 四. Substituting for $\phi_{e}$ and $\phi_{j}$ from Eq. (23) we obtain the following equation for the percolation line in the $(\phi, T)$ plane (cf. Fig. 1):

$$
\phi_{p}(T)=\frac{q}{6 \alpha} e^{\left(\epsilon_{j}-\epsilon_{e}\right) / T}
$$

The length scale $L=\bar{R}$ has no thermodynamic meaning and purely reflects a topological (infinite network vs. disconnected clusters) property. At infinite temperature, the junction-induced interaction becomes irrelevant and $\phi_{p}$ is the percolation threshold for the usual lattice percolation, studied in many classical works 24. The value $\phi_{p}=\frac{q}{6 \alpha}$ (equal to $\frac{1}{2(q-2)}$ for cubic lattice), obtained here is not inconsistent with the usual result $\phi_{p}=1 /(q-1)$ for the site percolation; the difference stems from the fact that in our model, there is percolation of two different objects: the monomers and the junctions, which do not occupy the sites of the same lattice; in our model, the monomers occupy the vertices of the lattice, while the junctions occupy the interstitial positions.

Although the percolation transition, where an infinite cluster is formed is not related directly to the junctions-ends transition discussed in Sec. 3.2, the junction induced interaction influences the shape of the percolation line at low temperatures, in agreement with earlier studies of the percolation in the interacting lattice gas [24]. The exact value of $\alpha$, and consequently of $\phi_{p}$, should not be taken too seriously, because the lattice approximation is not adequate for description of the system at the scale of an individual junction. Although, the calculation shows that the percolation line cuts the coexistence line of the phase transition on the left side of the critical point (cf. Fig. 1), in real physical systems, the intersection point can be also to the right of the critical point [35]. Rigid systems can have effectively different $\alpha$, as discussed in Sec6. Thus, depending on where the percolation line intersects the coexistence curve, in general, the coexisting phases discussed in Sec. 3 can be either two network phases, two phases of disconnected, branched clusters, or a network coexisting with dilute chains.

We now discuss the details of the cluster evolution as a function of monomer density $\phi$. Recall that the mean distance between the defects (i.e. 
junctions or ends)

$$
\bar{l} \simeq \frac{2 \phi}{\phi_{e}+3 \phi_{j}}
$$

is a non-monotonic function but has a maximum at $\phi_{e}=3 \phi_{j}$; this is precisely the condition that determines the percolation threshold. Noting that Eq.(49) implies that the cluster size increases monotonically with increasing density $\phi$, we conclude that there are two competing processes in cluster formation: the lateral growth and the 'filling' in from inside. As the monomer density is increased, for small densities up to the percolation threshold, $\phi=\phi_{p}(T)$, the clusters become larger but more sparse. At $\phi_{p}(T)$, an infinite cluster is formed and the process is reversed: clusters (including the infinite one) become denser, being predominantly filled in from inside. Although both below and above $\phi_{p}(T)$ the lateral size of the clusters increases, the rate of lateral growth with increasing monomer densities lower than the rate of internal densification above $\phi_{p}(T)$.

\section{$6 \quad$ Rigid and semiflexible chains}

Solutions of rigid and semiflexible chains present two other broad classes of systems which may show self-assembly and formation of ends and junctions, in a manner similar to that of flexible chains as discussed up to now. As an example, one can think about solutions or gels of actin, a biopolymer forming the cytoskeleton and involved in cell locomotion. Although it is difficult to introduce bending rigidity into a lattice model such as ours, the qualitative effects can be easily understood, at least at the mean field level. For flexible chains, as in our model, the lattice size is usually taken to be the persistence length (Kuhn segment for polymers), defining an effective 'monomer' size, while for semi-flexible chains, the lattice size would be the true size of molecular monomers, comprising the chains, the persistence length being much larger than the lattice size. On very general grounds, each defect (i.e. an end or a junction) contributes the energy $-k_{B} T$ to the free energy. Consequently, the defect-dependent part of the free energy in a system where non-conserved defects are present is

$$
F / T=-\phi_{\text {defects }}=-\phi_{e}-\phi_{j}
$$


in accord with Eq. (24) [45]. The difference between flexible chains and rigid ones lies in the dependence of $\phi_{e}$ and $\phi_{j}$ on the total monomer density $\phi$, which can be understood on the basis of the simple probabilistic argument outlined in Sec. 3.2. Two ends can join to form a bond. In the case of flexible chains any two ends which are neighbors on the lattice can form a bond, irrespective of the orientation of the adjacent links (see Fig. 2). In the case of rigid rods, only rods that are co-linear, can coalesce. Therefore, taking into account that the coalescence of two ends lowers the energy by an amount $2 \epsilon_{e}$, so that the Boltzmann factor for the probability of coalescence is proportional to $e^{2 \epsilon_{e}}$, we find that in equilibrium

$$
\begin{aligned}
\phi & =c \phi_{e}^{2} e^{2 \epsilon_{e}} \\
c & =q \text { flexible chains } \\
c & =1 \text { rigid rods }
\end{aligned}
$$

The same argument applies for a formation of a junction from three ends

$$
\begin{aligned}
\phi_{j} & =\alpha \phi_{e}^{3} e^{3 \epsilon_{e}-\epsilon_{j}} \\
\alpha & =q(q-2) / 3 \text { flexible chains (cubic lattice) } \\
\alpha & =1 \text { rigid rods }
\end{aligned}
$$

In general, for semiflexible chains $1 \leq c \leq q ; \alpha \leq q(q-2) / 3$. In the continuum limit $q \rightarrow 4 \pi$ for flexible chains. Therefore the most general form of the mean field free energy of Eq. (22) can be written:

$$
F / T \simeq(1-\phi) \ln (1-\phi)-\left(\frac{\phi}{c}\right)^{1 / 2} e^{-\epsilon_{e}}-\alpha\left(\frac{\phi}{c}\right)^{3 / 2} e^{-\epsilon_{j}}
$$

with $c$ and $\alpha$ depending on rigidity of the chains and of the junctions. In general, the degree of the flexibility of the junctions is not necessarily equal to that of the chains themselves. The former may depend on the microscopic properties of the cross-linker molecules as in the case of gels or on the properties of the surfactant molecules in the case of micelles and microemulsions, and other system-specific details.

The effect of the rigidity on the correlations is more difficult to treat quantitatively in the present framework due to formal difficulty of introducing the bending rigidity into the model. In particular, the influence of branching on the nematic transition, present in the solutions of rigid rods remains to be investigated. An heuristic theory [41] might be more useful for this purpose. 


\section{Discussion}

We have presented a generic model of self-assembling chains which can branch and form networks with branching points (junctions) of arbitrary functionality. The model rigorously maps the partition function of a solution of branched, self-assembling, mutually avoiding chains onto that of a Heisenberg magnet in the mathematical limit of zero spin components. The model has been studied in the mean field approximation, which neglects the presence of intracluster loops. It is found that despite the absence of any specific interaction between the chains, the presence of the junctions induces an effective attraction between the monomers, which in the case of three-fold junctions leads to a first order reentrant phase separation between a dilute phase consisting mainly of single chains, and a dense network. The reason for this entropic transition lies in the observation that although the translational entropy of the chains is lower in the dense phase, the total entropy is still higher due to the entropy of the large number of self-assembling junctions in the dense phase. The prerequisite for the phase separation is that the energy of a junction is lower than the energy a free end: $\epsilon_{j}<\frac{1}{3} \epsilon_{e}$, that is, the formation of junctions is energetically favorable (both $\epsilon_{e}, \epsilon_{j}$ are considered positive and are measured relative to the energy of a monomer in the middle of a chain). The model then modified in order to study topological properties of the system at the mean-field level. Independent of the junctions-ends transition, we predict the percolation (connectivity) transition at which an infinite network is formed. The percolation transition partially overlaps with the junctions-ends transition (cf. Fig. 1). This transition takes place at the point where the density of the free ends is three times the junctions density, $\phi_{e}=3 \phi_{j}$, which at the same time is the point where the mean distance between the defects (i.e., junctions or ends), $\bar{l}$ attains its maximum. This result agrees with other mean field level studies of percolation of tree-like clusters [4], [23]. This result means that up to the percolation transition the branched clusters grow laterally, predominantly by addition of the new branches at the periphery of a cluster, while at the percolation transition, an infinite network is formed and the process is reversed: the clusters grow mainly due to the filling of the internal mesh. The percolation transition is a continuous, non thermodynamic transition that describes a change in the topology of the system but not a thermodynamic phase transition. Our treatment which predicts both the thermodynamic phase equilibria as well as the spatial cor- 
relations in the system allows us to treat both the phase separation and the percolation threshold within the same framework, at least at the mean field level.

The predicted density-density correlation function has a usual OrnsteinZernicke form at low monomer densities. The correlation length of the density fluctuations $\xi$ is divergent at the spinodal line of the junctions-ends transition and is a non-monotonic function of the monomer density. $\xi$ attains its maximum around the point where the number of ends equals the number of junctions, $\phi_{e} \approx \phi_{j}$. It is important to emphasize that it is $\xi$ which is measured in actual scattering experiments, and not other lengths of the problem, such as the mean cluster radius $\bar{R}$ or the mean distance between the defects $\bar{l}$. The zero-wavevector scattering intensity, $S(0)$ also has a maximum as a function of $\phi$ along the same line, starting at the critical point. This effect is a direct consequence of the first-order phase separation between low and

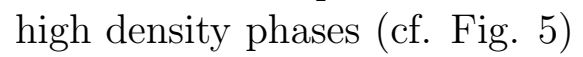

We also predict the emergence of the medium range correlations at high monomer and junction densities, as reflected in the predicted peak in the structure factor, signifying the onset of structural correlations in the system (cf. Fig. 5).

The theory presented here has many physical realizations. We now discuss the implications of the generic results for several specific cases.

\subsection{Physical gels.}

Physical gels include a broad class of systems, consisting of long polymer chains that are reversibly cross-linked. The cross-links can break and reform under the influence of thermal fluctuations. Examples of these materials are numerous, many of them of practical interest [26]. The chains that comprise the basis of the gel, can either be self-assembling or chemically bonded [22]. With increasing monomer density, the gelation threshold is reached where a connected network (gel) is formed. Despite intensive research in the past two decades, several features of the physical gelation process are still under debate. One question concerns whether the gelation transition (i.e., the transition from disconnected, branched clusters to a macroscopic, connected network) is a structural transition or a thermodynamic one. Another question of interest is: if the gel transition is indeed a thermodynamic one, what is the order of the transition [26, 36]. Our results show that actually there are two independent transitions. The first one is the classical percolation transition, 
located at the point where the density of the cross-links is equal to one third of the density of the free ends in agreement with classical result due to Flory [4]. An infinite connected cluster is formed at the percolation threshold. This transition is structural and is not connected to any thermodynamic singularity.

For three-fold cross-links another, a thermodynamic transition is also present at small densities, and partially overlaps the percolation line (see Fig. 1). This transition is the first order phase transition due to inter-monomer attraction induced by the presence of the junctions and not due to the aggregation of stickers (cross-link molecules). Our model shows that the phase separation can occur under good solvent conditions, and not only in $\Theta$-solvents. An advantage of the present model is that it starts from a rigorous description of a solution of self-avoiding branched chains and does not involve any ad hoc hypotheses about interactions between the monomers, cross-linkers and solvent molecules. An interesting problem is the difference between gelation, when molecular segments are allowed to polymerize and cross-link simultaneously in the reaction bath, and vulcanization, when preexisting polymer chains are cross-linked by irradiation, addition of linker molecules, or other means. In addition to the fact that one expects large spatial inhomogeneities in the case of vulcanization [27], we argue that an additional major difference is the functionality of the cross-links. In the case of gelation, most of the junctions will be three-fold, simply due to the fact that the collision of three segments is more probable than a collision of four. In the case of vulcanization, on the contrary, the junctions will be four-fold, being formed by cross-linking two pre-existing chains. So, in certain cases gelation will be observed experimentally as a first-order thermodynamic transition, while vulcanization is always continuous non-thermodynamic transition. As a matter of fact, we have shown that the presence of four-fold junctions is thermodynamically equivalent to decreasing the quality of the solvent, in accord with the results obtained by other theoretical methods [27] and Monte Carlo simulations [26].

The long-standing question about the presence of closed, intra-cluster loops in the pre-gel clusters and in the gel phase, becomes irrelevant in the present formulation. In our model, nothing prevents the intra-cluster loops from forming and their number is determined by the distributions of ends and junctions. However, we note that the question of the influence of the loops on the thermodynamic and structural properties cannot be addressed properly in the mean field approximation, used in the paper, because it effectively 
disregards the loops.

Experimentally, the gelation transition is commonly observed by measuring a viscosity increase due to the formation of the macroscopic network. Therefore, the nature of the experimentally observed gelation transition will depend on the path in the phase space along which the transition is approached. For example, decreasing the temperature at constant monomer density along the arrow $b$ of Fig.1 will be produce a continuous, non-thermodynamic gelation transition at the point where line $b$ intersects the percolation line. On the contrary, a temperature decrease along the arrow $a$ will produce a first order phase separation between a phase of dilute chains and a connected network (gel).

\subsection{Microemulsions}

Microemulsions consist of domains of two immiscible fluids (typically, water and oil) stabilized by a surfactant [3, 5]. One theoretical approach to their understanding is to map the oil-in-water dispersion onto a lattice model in the following manner. When the spontaneous curvature [6] is small, the relevant length scale is the persistence length of the surfactant film; this length is determined by the bending rigidity and the thermal fluctuations [44. For systems where the spontaneous curvature dominates (this occurs when the spontaneous curvature is larger than the inverse of the persistence length), for a certain range of spontaneous curvatures, one finds that the oil or water domains are either spheres or elongated tubes of radius $r \sim c_{0}^{-1}$. For oil internal systems, the radius of the oil domains can also be kept constant by maintaining fixed ratio of the surfactant density to that of oil $\frac{\phi_{\text {surf }}}{\phi_{\text {oil }}}$ (in a water internal system it is given by the surfactant to water ratio). The application of the general results of this paper to these cases requires that one understands the mapping between the microemulsion and our lattice model. Taking $r$ as the lattice constant, and considering an oil internal system, the microemulsion can be mapped to a solution of self-assembling, branched chains (oil domains $\rightarrow$ 'monomers'; oil+surfactant fraction $\rightarrow \phi$ ) as described by our model. Obviously, the analogy breaks down at oil volume fractions close to one, because our model does not allow the merging of a two elongated thin adjacent domains into a wider one.

Using this analogy, the evolution of spontaneous curvature dominated microemulsions can be qualitatively described in the context of our model and the results presented above, as follows. At low oil fraction, the microemulsion 
consists of spherical droplets of radius $r \sim c_{0}^{-1}$. At a certain oil fraction a 'polymerization transition' [20, 42] takes place and cylinders of radius $r$ are formed which eventually branch. With increasing oil fraction at a certain oil fraction $\phi_{p}(T) \simeq$ const $\times e^{\left(\epsilon_{e}-\epsilon_{j}\right) / T}$, a percolating oil domain is formed, indicating the onset of the bicontinuous microemulsion (cf. Sec. 4.1.2). At low temperatures, this transition is masked by a first order, phase separation transition due to junction-induced attraction between the oil domains (Sec. 3.2). This first-order transition is present only if the branching points are three-fold, which seems to be relevant to microemulsions. In addition to the fact that three-fold branches are more probable statistically, the energy of the three-fold junctions is lower than that of four- and higher fold junctions, as can be shown from calculations of the bending energy of the surfactant bilayers in the junction region [42].

The structure factor (for scattering from the bulk water or oil domains i.e., bulk contrast experiments) has a simple Ornstein-Zernicke form in the droplet microemulsion region (which includes also elongated but disconnected cylindrical droplets) with a correlation length that diverges at the spinodal line of the first-order transition. In the bicontinuous region, the structure factor is an increasing function of the wavevector, as expected in any high density system (cf. Sec.4.1.2). With increase in the number of junction, either due to increase in the monomer density, or the temperature concentration a peak appears in the structure factor, indicating the emergence of medium range correlations between oil domains (cf. Fig. 5). The peak is predicted to appear whenever the condition $\phi \gtrsim \frac{\alpha}{2 q} e^{-2 \epsilon_{3} / T}$ is satisfied. The peaked structure factor is a distinctive characteristic of bicontinuous microemulsions, as borne out by many experimental studies [5, 43]. It is important to emphasize, that our model predicts that the peak occurs only when $\phi>\max \left[\frac{1}{2}, \phi_{b}\right]$ which means that, in principle there may be network microemulsions that $d o$ not show a peak in the structure factor ( the region between the percolation line and vertical $\phi=1 / 2$ line Fig. 5). The fact that the peak appears only for $\phi>1 / 2$ should not be taken too seriously. The exact value $\phi=\frac{1}{2}$ is most probably an artifact of a lattice construction, reflecting the monomer-hole symmetry. More important is the qualitative prediction that the peak in the structure factor is a consequence of the correlation between the oil domains, induced by the presence of the junctions. 


\subsection{Wormlike micelles}

Surfactant molecules in aqueous solutions can form long, cylindrical micelles in certain regions of the phase diagram. In the cylindrical phase, each micelle consists of a large numbers of surfactant molecules. The micelles are polydisperse in size, the equilibrium distribution being determined by the interplay between the entropy (which favors small micelles) and the 'cap' energy, which is determined by the geometry of packing of surfactant molecules at the ends of a micelle. Due to the bending rigidity of the surfactant layer, the micelles are stiff up scales of order of the persistence length $l_{p}$ [6]. On the other hand, for length scales much larger than $l_{p}$, the micelles can be considered as flexible chains. Therefore, for long enough micelles $\left(h_{0} / \sqrt{2 \phi} \ll 1\right)$, the lattice constant of the equivalent Heisenberg model can be taken to be equal to the persistence length $l_{p}$. In the opposite case of short chains, on can take a surfactant molecule as a 'monomer' size, with the reservations described in Sec. 6.

It has been suggested on the basis of rheological, conductivity and dielectric polarizability experiments, that the micelles can branch, and at a certain point the system transforms to a connected micelle network. The energy of the junctions and the ends in this system can be varied by changing the salt concentration. Theoretically, it was proposed that the ' $n=0$ ' model might describe the formation of a network of wormlike micelles [31, 32]. Our results substantiate these suggestions and indeed predict the formation of a connected micellar network, which can partially overlap with the first order, phase separation phase transition.

Results of neutron scattering performed on wormlike micelles solutions show that the correlation length, $\xi$, of the density fluctuations is a nonmonotonic function of the density in the proposed branched state [33], which might be explained by the results of Sec. 4.1.1. These experimental results were interpreted in Ref. [33] in terms of the mean micelle size (analogous to $\bar{l}$ in our formulation). However, it is important to realize that, although both $\xi$ and $\bar{l}$ exhibit a maximum as a function of the density, it is $\xi$ which is actually measured in scattering experiments, while $\bar{l}$ conveys purely geometrical information, not directly measured in scattering experiments. The correlation length $\xi$ has a maximum around $\phi_{e}=\phi_{j}$ which is a direct consequence of the junctions-ends transition, discussed in Sec. 3.2. It should be noted that the maximum of $\xi$ is not directly related to the network formation, which takes place at $\phi_{e}=3 \phi_{j}$, as well as $\bar{l}$. 


\subsection{Dipolar and magnetic fluids and colloids}

Dipolar fluids or colloids consist of dipolar particles, bearing electric or magnetic dipole. For, example ferrofluids. consist of magnetic (metallic) particles immersed in an inert fluid. The molecules of real liquids, such as acetone $\left(\mathrm{C}_{3} \mathrm{H}_{6} \mathrm{O}\right)$ or dimethylsulfoxide $\left(\mathrm{C}_{2} \mathrm{H}_{3} \mathrm{SO}\right)$ also bear electronic dipolar moments. The interaction energy of two dipoles has a minimum when the dipole moments are collinear [11]. This means that dipolar particles have a tendency to aggregate into chains [46], so that all the dipole moments of the particles in the chain are more or less colinear. However, it is important to realize that these chains can also branch [45]. The energy of the particles at the branching point is obviously higher than in the middle of a chain and can be calculated form electrostatics (or magnetostatics in the case of magnetic dipoles) 45]. Disregarding the long-range part of the dipole-dipole interactions, the system of both finite ended and branched dipolar chains can be mapped to the model presented in this paper and our predictions for the phase separation, scattering and percolation would apply. This mapping is justified in dilute systems such as ferrofluids. or magnetic colloids. In dense molecular liquids, it probably breaks down. In order to understand the properties of molecular dipolar liquids, one may have to take into account specific interactions between the molecules, such as hydrogen bonding.

\subsection{Actin networks}

Actin is a self-assembling biopolymer, which supports the membranes of biological cells. Sol-gel transition of actin solutions and networks, induced by various proteins, (e.g., myosin) plays an important role in cell movement and locomotion. The properties of actin networks in vivo have been studied extensively by biochemical and molecular biology methods [9]. Recently, physical properties of actin solutions and gels have been studied in vitro, in rheological and scattering experiments 44]. The experimentally obtained phase diagram is strikingly similar to Fig. 1, where at the percolation line there is a transition from and entangled network to a connected 'microgel' state. However, it was observed that at low temperatures the dense phase at the coexisting curve consists of 'bundles', where several parallel actin filaments are tightly bound together by the cross-links [48].

Actin filaments are rigid, with the persistence length as high as ten microns. However, the general results of this paper should apply, with the 
reservations made in Sec. 6, which accounts for the similarity between the predicted phase diagram and the experimentally found one. The formation of the bundles is probably due to nematic interaction between rigid filaments and cannot be captured in the present model. However, one can estimate the condition for the bundles formation using a crude qualitative argument. For this purpose, the network phase can be viewed as a collection of rigid filaments of average length $\bar{l} \simeq e^{\epsilon_{j} / T} \phi^{-1 / 2}$ (cf. Sec.3.1.1). The orientational order appears in the solutions of rigid rods when $\phi \gtrsim$ const $/ L$, which means that for $\phi \gtrsim e^{-2 \epsilon_{j} / T}$ one might expect appearance of the bundles.

Acknowledgment The authors thank R. Granek, J.-F. Joanny, T. Lubensky, S. Panyukov, P. Pincus, Y. Rabin, E. Sackmann, T. Tlusty and T. Witten for helpful discussions and the anonymous referee for illuminating remarks. The support from ISF Center of Excellence on Self-Assembly, the ACS and the donors of the Petroleum Research Fund and Schmidt-Minerva Center is gratefully acknowledged.

\section{Appendices}

\subsection{AppendixA: ' $n=0$ ' model}

The formal aspects of the ' $n=0$ ' model have been studied extensively 11 , 12, 19. For the sake of completeness we review the derivation here. Let us calculate the generating function $\operatorname{Tr} e^{\vec{k} \vec{s}}$ for an $n$-component spin, $\vec{s}$, on a $d$-dimensional lattice, where the averaging is over solid angle of the vector $\vec{s}$, subject to condition $\sum_{\alpha} s_{\alpha}^{2}=n$.

$$
\operatorname{Tr} e^{\vec{k} \vec{s}}=\frac{\int \prod_{\alpha} d s_{\alpha} \delta\left(\sum_{\alpha} s_{\alpha}^{2}-n\right) e^{\vec{k} \cdot \vec{s}}}{\int \prod_{\alpha} d s_{\alpha} \delta\left(\sum_{\alpha} s_{\alpha}^{2}-n\right)}=\frac{g_{n}(\vec{k})}{g_{n}(0)}
$$

The cumulants of $\vec{s}$ can be obtained by differentiating the generating function with respect to $k$. Now, supposing that $\vec{k}=(k, 0, \ldots, 0)$

$$
\begin{gathered}
g_{n}(k)=\int \prod_{\alpha} d s_{\alpha} \delta\left(\sum_{\alpha} s_{\alpha}^{2}-n\right) e^{\vec{k} \cdot \vec{s}}=\int_{-\infty}^{\infty} d \omega e^{i \omega n} \int d s_{1} e^{k s_{1}-i s_{1}^{2} \omega} \int \prod_{\alpha=2}^{n} d s_{\alpha} e^{-i \omega s_{1}^{2}}= \\
=\pi^{n / 2} \int_{-\infty}^{\infty} d \omega \frac{e^{i \omega n+\frac{k^{2}}{4 i \omega}}}{(i \omega)^{n / 2}}=\pi^{n / 2} i^{-n / 2}\left[\int_{0}^{\infty} d \omega \frac{e^{i n\left(\omega-\frac{k^{2}}{4 n \omega}\right)}}{\omega^{n / 2}}+(-1)^{n / 2} \int_{0}^{\infty} d \omega \frac{e^{-i n\left(\omega-\frac{k^{2}}{4 n \omega}\right)}}{\omega^{n / 2}}\right.
\end{gathered}
$$


The integrals in step two of the above equations, although formally divergent, should be treated as generalized functions. The resulting integrals are known [7]. Putting $\nu=1-n / 2$, we find

$$
\begin{aligned}
g_{n}(k) & =\pi^{n / 2} i^{-n / 2} 2\left(\frac{\pi i}{2}\right)\left(\frac{k}{2 \sqrt{n}}\right)^{\nu}\left[H_{-\nu}^{(1)}(i k \sqrt{n})+H_{-\nu}^{(2)}(i k \sqrt{n})\right]= \\
& =\pi^{n / 2} i^{-n / 2} 2 \pi i\left(\frac{k}{2 \sqrt{n}}\right)^{\nu} e^{-i \frac{\nu \pi}{2}} I_{-\nu}(k \sqrt{n})=\pi^{\frac{n}{2}+1} n^{\frac{n}{2}-1} \frac{I_{\frac{n}{2}-1}(k \sqrt{n})}{(k \sqrt{n} / 2)^{\frac{n}{2}-1}}
\end{aligned}
$$

where $H_{p}^{(1)}(x), H_{p}^{(2)}(x)$ are Hankel functions of the order $p$, and $I_{p}(x)$ is a modified Bessel function of the order $p$. Now, up to second order

$$
I_{p}(x)=\left(\frac{x}{2}\right)^{p}\left(\frac{1}{\Gamma(p+1)}+\frac{1}{2 \Gamma(p)} x^{2}+O\left(x^{4}\right)\right)
$$

Keeping in mind that in our case $p=\frac{n}{2}-1$, it follows

$$
\lim _{n \rightarrow 0} \operatorname{Tr} e^{\vec{k} \vec{s}}=\lim _{n \rightarrow 0} \frac{g_{n}(k)}{g_{n}(0)}=1+\frac{1}{2} k^{2}
$$

In particular,

$$
\operatorname{Tr} s_{\alpha}^{m}=\frac{d^{m}}{d k^{m}}\left[\lim _{n \rightarrow 0} \operatorname{Tr} e^{\vec{k} \vec{s}}\right]_{k=0}=\delta_{m, 2} \delta_{\alpha, 1}
$$

and

$$
\operatorname{Tr} s_{\alpha} s_{\beta}=\frac{\partial^{2}}{\partial k_{\alpha} \partial k_{\beta}}\left[\lim _{n \rightarrow 0} \operatorname{Tr} e^{\vec{k} \vec{s}}\right]_{k=0}=\delta_{\alpha, 1} \delta_{\beta, 1}
$$

Due to the fact that the generating function has only quadratic terms in $k$, cumulants higher than second order vanish. This is because the higher order cumulants are related to higher order derivatives of the generating function with respect to $k$ and these all vanish, as seen from Eq. (51)

\subsection{Appendix B: monomers, bonds and junctions on a lattice}

\subsubsection{Relation between the densities}

The chains consist of monomers, occupying the vertices of the lattices, connected by bonds. Each junction is not occupied by a monomer but is formed 
by three unphysical 'bonds', connecting three adjacent monomers as illustrated in Fig. 2. The bonds forming the junction are not counted by the model. The monomers comprising a chain fall into two categories: internal ones, attached to two bonds, and the monomers at the ends of the cluster, attached only to one bond. The total number of bonds (including the unphysical ones) is therefore

$$
N_{\text {bonds }}^{\text {total }}=\frac{1}{2}\left(2 N_{i}+N_{e}+3 N_{j}\right)
$$

where $N_{i}$ is the number of internal monomers, $N_{j}$ is the number of junctions, and $N_{e}$ is the number of ends; the prefactor $\frac{1}{2}$ accounts for the double counting of each bond. The total number of monomers is

$$
N=N_{i}+N_{e}
$$

and the number of physical bonds, connecting a pair of monomers, is the total number of bonds less those unphysical bonds which are involved in junctions

$$
N_{\text {bonds }}=N_{\text {bonds }}^{\text {total }}-3 N_{j}
$$

Combining these equations together, one gets

$$
N=N_{\text {bonds }}+\frac{1}{2} N_{e}+\frac{3}{2} N_{j}
$$

\subsubsection{Value of $\alpha$}

Here we present the considerations which lead to particular values of the constants in mean-field equations of Sec. 3. In our model we consider each site of a lattice occupied either by a monomer or solvent molecule. Chain bonds are defined as the bond between two occupied neighbor sites. A junction can be formed when three ends are neighbors on a lattice as shown in Fig. 2. The sum in the three-spin term $\sum_{i j k} K S_{i} S_{j} S_{k}$ in Eq. (18), accounting for the junctions between the chains, is constrained over all distinct triplets of spins $i, j, k$. Tthe three-spin term can be rewritten as $\frac{1}{3} \sum_{i} K S_{i} \sum_{j k}^{(K)} S_{j} S_{k}$ where $\sum_{i}$ is now an unconstrained summation over all cites and $\sum_{j, k}$ is unconstrained sum over all possible pairs of sites $j, k$ which together with $i$ form a junction $i, j, k$. In the mean field approximation this term is fuirther 
Figure 7: Two possible configurations of the triplet $i, j, k$ : a) the $j$ and $k$ sites are the nearest neighbors of the site $i$; b) $k$ is the nearest neghbor of the site $i$ while $j$ is the next nearest neighbor of $i$.

transformed into $\frac{1}{3} \sum_{i} 3 K S_{i} \sum_{j k}^{(K)}\left\langle S_{j}\right\rangle\left\langle S_{k}\right\rangle$. On a hypercubic lattice, there are two possible configurations of the triplet $i, j, k$. The first one, is shown in Fig. $1(\mathrm{a})$ : both $j$ and $k$ are the nearest neigbors of the site $i$. The second is realized when either $j$ or $k$ is a next nearest neighbor of $i$, shown in Fig. 7(b). A simple calculation shows that for a hypercubic lattice of a coordination number $q$, the number of such pairs of the first kind is is $q(q-2)$ and the number of the pairs fo the second kind is $\frac{1}{2} q(q-2)$. Therefore, $\alpha=\frac{1}{3}\left(q(q-2)+\frac{1}{2} q(q-2)\right)=\frac{1}{2} q(q-2)$. For a hexagonal close-packed structure or for the f.c.c. lattice, a similar calculation gives $\alpha=q(\mathbb{B}(\mathrm{a}))$. However, the exact numerical value of $\alpha$ is an artefact of the lattice construction and should be thought of as a phenomenological parameter reflecting the microscopic features of a junction, along with the junction energy $\epsilon_{j}$. A particular value of $\alpha$ affects only the numerical values of the critical temperature and density but not the overall qualitative behavior. It can be adjusted to reflect physically allowable junction configurations. For example, three neigboring chains, coming perpendicular to the plane of a triplet $i, j, k$ and connected by it, can hardly be interpreted as a junction in most of the experimental systems. For convenience, we have used $\alpha=\frac{1}{3} q(q-2)$ in calculating the phase diagram and the structure factor. 
Figure 8: Two possible alternative definitions of a junction. a) shows a junction on a hexagonal lattice, $\alpha=q / 2 ; b)$ shows a larger junction, coupling the sites separated by two lattice constants.

\subsection{Appendix C: density-density correlations}

In this Appendix we show the derivation of the result (36) of Sec. \$ for the density-density correlation function. From Eq.(33)

$$
\begin{aligned}
s_{i} & =\frac{x_{i}}{1+\frac{1}{2} x_{i}^{2}} \\
x_{i} & =\sqrt{2 \phi_{i} /\left(1-\phi_{i}\right)} ; \frac{\partial \ln x_{i}}{\partial \phi_{k}}=\delta_{i k} \frac{1}{2 \phi_{i}\left(1-\phi_{i}\right)} \\
s_{i} & =\sqrt{2 \phi_{i}\left(1-\phi_{i}\right)} ; \frac{\partial s_{i}}{\partial \phi_{k}}=\delta_{i k} \frac{1-2 \phi_{i}}{\sqrt{2 \phi_{i}\left(1-\phi_{i}\right)}} \\
J_{i} & =\frac{x_{i}-h_{i}-K_{i} \sum_{j, k}^{(K)} s_{j} s_{k}}{\sum_{j} s_{j}}
\end{aligned}
$$

Consequently,

$\ln J_{i}=\ln x_{i}-\ln \sum_{j} s_{j}-\frac{h_{0}}{\sqrt{x_{i} \sum_{j} s_{j}}}-3 K_{0} \frac{\left(\frac{1}{3} \sum^{(K)} s_{j} s_{k}\right) x_{i}^{1 / 2}}{\left(\sum_{j} s_{j}\right)^{3 / 2}}+O\left(K_{0}^{2}, h_{0}^{2}, K_{0} h_{0}\right)$ 
Now,

$$
\begin{aligned}
\left\langle\delta \phi_{i} \delta \phi_{k}\right\rangle= & \frac{\partial \ln J_{i}}{\partial \phi_{k}}=\frac{\partial \ln x_{i}}{\partial \phi_{k}}-\frac{\sum_{j} \frac{\partial s_{j}}{\partial \phi_{k}}}{\sum_{j} s_{j}}+\frac{h_{0}}{2\left(x_{i} \sum_{j} s_{j}\right)^{3 / 2}}\left[\frac{\partial x_{i}}{\partial \phi_{k}} \sum_{j} s_{j}+x_{i} \sum_{j} \frac{\partial s_{j}}{\partial \phi_{k}}\right] \\
& -3 K_{0}\left(\frac{1}{3} \sum_{j p}^{(K)} s_{j} s_{p}\right)\left[\frac{1}{2 x_{i}^{1 / 2}\left(\sum s_{j}\right)^{3 / 2}} \frac{\partial x_{i}}{\partial \phi_{k}}-\frac{3}{2} \frac{x_{i}^{1 / 2}}{\left(\sum_{j} s_{j}\right)^{5 / 2}} \sum_{j} \frac{\partial s_{j}}{\partial \phi_{k}}-\right. \\
& -3 K_{0} \frac{x_{i}^{1 / 2}}{\left(\sum_{j} s_{j}\right)^{3 / 2}} \frac{2}{3}\left(\sum_{j p}^{(K)} s_{p} \frac{\partial s_{j}}{\partial \phi_{k}}\right)
\end{aligned}
$$

Together with Eq.(52) this leads to Eq. (36) of Sec.4.1.

\subsection{Appendix D: spin-spin correlations}

This Appendix shows the detailed calculation of the spin-spin correlation function of Sec. 5. In principle, the scalar products of any two spins in Eq. (10) contain both longitudinal component, $S_{1}$, parallel to the direction of the field $\vec{h}$, and a 'transverse' one, $\vec{S}_{\perp}$. For the two spin term it gives

$$
J \vec{S}_{i} \cdot \vec{S}_{j}=J\left(S_{1, i} S_{1, j}+\vec{S}_{i, \perp} \cdot \vec{S}_{j, \perp}\right)
$$

and the three-spin term is chosen to be

$K \sum_{i j k}\left(S_{1, i} S_{1, j} S_{1, k}+\frac{\beta}{3}\left[S_{1, i}\left(\vec{S}_{\perp, i} \cdot \vec{S}_{\perp, k}\right)+S_{1, j}\left(\vec{S}_{\perp, i} \cdot \vec{S}_{\perp, k}\right)+S_{1, k}\left(\vec{S}_{\perp, i} \cdot \vec{S}_{\perp, j}\right)\right]\right)$

The average value of the 'transverse' component, $\left\langle\vec{S}_{\perp}\right\rangle$, is equal to zero, because the average magnetization is aligned along the field $\vec{h}$. Consequently, it has no consequence for the thermodynamic properties, and was neglected in the calculation of the free energy in the Sec. 3. However, its fluctuations around its average of zero, are not zero, and convey physically important information. Neither $S_{1}$ nor $\vec{S}_{\perp}$ has any direct physical meaning. However, physically relevant information can be extracted from the knowledge of their correlation functions. 
Consider the correlation function $\left\langle S_{1, i} S_{1, j}\right\rangle$ between the 'longitudinal' components of two spins located at sites $i$ and $j$. As follows from the discussion of the Secs. 2,2.2 the only terms entering its expansion $\operatorname{Tr} S_{1, i} S_{1, j}$ $\mathrm{e}^{-H\left\{S_{k}\right\} / T}$ are the same as of Eq. (13) with $S_{1, i} S_{1, j}$ replacing the $h$-terms $h S_{i}$ and $h S_{j}$. This means that the correlator $\left\langle S_{1, i} S_{1, j}\right\rangle$ counts all the configurations where there are chain ends at the point $i$ and at the point $j$. In other words, $\left\langle S_{1, i} S_{1, j}\right\rangle$ measures the correlations between any two ends. Recalling that the ends density is $\phi_{e}\left(r_{i}\right)=h_{i} S_{1, i}$, and subtracting the $\delta_{i j}$ contribution corresponding to the correlation of an end with itself, we arrive at Eqs. (46) (48), which can also be obtained directly from the relation

$$
\left\langle\delta \phi_{e}\left(r_{i}\right) \delta \phi_{e}\left(r_{j}\right)\right\rangle=\frac{\partial \phi_{e}\left(r_{i}\right)}{\partial h_{j}}
$$

To calculate the 'transverse' correlation function, $\left\langle S_{i, \perp} S_{j, \perp}\right\rangle$, where now $S_{\perp}$ signifies a component of $\vec{S}$ in one of the $(n-1)$ 'transverse' directions, one must focus on the second term of Eq.(53). Following the arguments of Sec. 2.2, the only terms which enter into expansion of $\left\langle S_{i, \perp} S_{j, \perp}\right\rangle$ in the limit $n \rightarrow 0$ are of the form

$$
\begin{aligned}
& \mathrm{S}_{i, \perp}[J S_{i, \perp} S_{m, \perp} J S_{k^{\prime}, \perp} S_{k, \perp} \underbrace{\left[\frac{\beta}{3} K S_{m, \perp} S_{n, 1} S_{k, \perp}\right]}_{\text {junction }} J S_{n, 1} S_{n^{\prime}, 1} \ldots \\
& \left.\ldots K S_{m^{\prime}, 1} S_{p, 1} S_{q, 1} \ldots . . J S_{j^{\prime}, \perp} S_{j, \perp} h S_{q, 1}\right] \mathrm{S}_{j, \perp}
\end{aligned}
$$

that is, the clusters, consisting of the 'backbone' of the $S_{\perp}$ 's with branched 'sidechains' of the $S_{1}$ 's, closed by $S_{i, \perp}$ and $S_{j, \perp}$. Inspection of possible terms of the form (55) shows Eq.(53)that the sites $i$ and $j$ must belong to the same chain, because the Eqs. (53,54) couple either two transverse components or a longitudinal one with two transverse. In other words, the 'transverse' correlator measures the correlations between any two ends belonging to a same chain. It is seen from equation (55) that the junctions enter the expansion of the transverse correlator with the weight $\frac{\beta}{3} K$. Since one wants to measure the correlations between the ends of a cluster belonging to the expansion of the original Hamiltonian (10), where all the junctions enter with the same weight $K$, the value $\beta=3$ must be chosen. That is, the relevant part of the three-spin term in the Hamiltonian (10) has the form

$$
K \sum_{i j k}\left(S_{i}^{1} S_{j}^{1} S_{k}^{1}+S_{i}^{1}\left(S_{i}^{\perp} S_{k}^{\perp}\right)+S_{j}^{1}\left(S_{i}^{\perp} S_{k}^{\perp}\right)+S_{k}^{1}\left(S_{i}^{\perp} S_{j}^{\perp}\right)\right)
$$


It is important to emphasize that the model given by Eq. (54) is suitable only for loopless clusters. Clusters containing loops enter the expansion with wrong weights, due to summation over $n-1$ 'transverse' components. However, the clusters with loops are neglected in the mean field approximation and one can use the model given by Eq.(54).

Performing the mean field approximation on these terms, as outlined in Sec. 8.3 gives for the local spin averages :

$$
\begin{aligned}
s_{i, 1} & =\frac{\partial \ln Z}{\partial h_{i, 1}}=\frac{x_{1}}{1+\frac{1}{2}\left[x_{1}^{2}+x_{\perp}^{2}\right]} \\
s_{i, \perp} & =\frac{\partial \ln Z}{\partial h_{i, \perp}}=\frac{x_{\perp}}{1+\frac{1}{2}\left[x_{1}^{2}+x_{\perp}^{2}\right]}
\end{aligned}
$$

where

$$
\begin{aligned}
& x_{1}=h_{i, 1}+\sum_{j} J s_{j, 1}+3\left(\frac{1}{3} \sum_{j, k}^{(K)} K s_{j, 1} s_{k, 1}\right) \\
& x_{\perp}=h_{i, \perp}+\sum_{j} J s_{j, \perp}+6\left(\frac{1}{3} \sum_{j, k}^{(K)} K s_{j, \perp} s_{k, 1}\right)
\end{aligned}
$$

with $\sum_{j}$ is the sum over nearest neighbors of the site $i$ and $\sum_{j, k}^{(K)}$ is the sum over the pairs of sites $j, k$ which together with $i$ make the junction triplet $i, j, k$ as in Sec. 5. The coupling between the parallel and transverse components of $s$ in the three-spin term in Eq.(56) has no consequence for the thermodynamic properties because the average value of 'transverse' component is zero: $\left\langle S_{\perp}\right\rangle=s_{\perp}=0$. The terms containing products $s_{i, \perp} s_{j, \perp}$ have been omitted for the same reason. However, this coupling is important for correlations, as we shall see. We thus define the spin-spin correlation function $C_{i k}^{(a)}$, whose 'longitudinal' component is given by:

$$
\begin{aligned}
C_{i k}^{(1)} \equiv & \left\langle S_{i, 1} S_{k, 1}\right\rangle-s^{2}=\frac{\partial s_{i, 1}}{\partial h_{k, 1}}=A^{(1)}\left[\delta_{i k}+J \sum_{j \in n n_{i}} \frac{\partial s_{j, 1}}{\partial h_{k, 1}}\right. \\
& \left.+6 K\left(\frac{1}{3} \sum_{m, j}^{(K)} s_{m, 1} \frac{\partial s_{j, 1}}{\partial h_{k, 1}}\right)\right] \\
\text { with } A^{(1)}= & \left.\frac{\partial}{\partial x_{1}}\left(\frac{x_{1}}{1+\frac{1}{2}\left[x_{1}^{2}+x_{\perp}^{2}\right]}\right)\right|_{x_{\perp}=0}=\frac{1}{1+\frac{1}{2} x_{1}^{2}}-\frac{x_{1}^{2}}{\left(1+\frac{1}{2} x_{1}^{2}\right)^{2}}
\end{aligned}
$$


For transverse component, (bearing in mind that $\left\langle S_{\perp}\right\rangle=s_{\perp}=0$ ) we obtain:

$$
\begin{aligned}
C_{i k}^{(\perp)} \equiv & \left\langle S_{i, \perp} S_{k, \perp}\right\rangle=\frac{\partial s_{i, \perp}}{\partial h_{k, \perp}}=A^{(\perp)}\left[\delta_{i k}+J \sum_{j \in n n_{i}} \frac{\partial s_{j, \perp}}{\partial h_{k, \perp}}\right. \\
& \left.+6 K\left(\frac{1}{3} \sum_{m, j}^{(K)} s_{m, 1} \frac{\partial s_{j, \perp}}{\partial h_{k, \perp}}\right)\right] \\
A^{(\perp)}= & \left.\frac{\partial}{\partial x_{\perp}}\left(\frac{x_{\perp}}{1+\frac{1}{2}\left[x_{1}^{2}+x_{\perp}^{2}\right]}\right)\right|_{x_{\perp}=0}=\frac{1}{1+\frac{1}{2} x_{1}^{2}}
\end{aligned}
$$

Note that the coupling between $s_{\perp}$ and $s_{1}$ in Eq.(58) does contribute to the transverse spin-spin correlation function. Substituting from Eq.(B3) one finds

$$
\begin{aligned}
A^{(1)} & =(1-2 \phi)(1-\phi) \\
A^{(\perp)} & =(1-\phi)
\end{aligned}
$$

Recalling that $\sum_{m, j}^{(K)}=\frac{2}{3} \frac{\alpha}{q} \sum_{n n_{i}}+\sum_{n n n_{i}}$, after Fourier transform $C_{i k}^{(a)}=$ $\sum_{\vec{q}} e^{i \vec{p}\left(\vec{r}_{i}-\vec{r}_{k}\right)} C_{\vec{p}}^{(a)}(a=\{1, \perp\})$ the equations (57) and (58) yield the Eq. (47) of Sec. 5 . It is important to emphasize that the model given by Eq. (54) is suitable only for loopless clusters. Clusters containing loops enter the expansion with wrong weights, due to summation over $n-1$ 'transverse' components. In principle, one can include higher order terms, coupling different 'transverse' components. However, as seen from the calculation above, in the mean field approximation, these terms do not contribute either to the free energy or to the spin-spin correlation function. That is, the mean field results are independent of the precise way in which the weights are assigned to clusters with loops. It follows that the mean field approximation disregards the loops, which contain information about long range intra-cluster correlations. Only the tree-like clusters give contribution to the mean field approximation. It is also possible to show by direct calculation from the rigorous Lubensky-Isaacson model that in the mean field approximation the number of loops vanishes.

\subsection{Appendix E: Exact model for percolation}

In order extract the information about the topological structure of the system, while retaining the exact correspondence between the expansion terms of the spin model, and the equilibrium branched clusters, one has to resort to 
tensor order parameter or, equivalently, to use several coupled $n=0$ fields. The presentation in this section follows closely the derivation of Ref. 23] with the major distinction that the junctions are not treated as point-like objects, which allows one to use a conventional normalization of the $n=0$ spins $\vec{S}$. The Hamiltonian to be used is

$$
H_{T}=h \sum_{i} \sum_{\alpha=1}^{m} S_{1, i}^{\alpha}+J \sum_{\langle i, j\rangle} \sum_{\alpha=1}^{m} \vec{S}_{i}^{\alpha} \vec{S}_{j}^{\alpha}+K \sum_{\langle i, j, k\rangle} \sum_{\alpha=1}^{m} S_{1, i}^{\alpha} S_{1, j}^{\alpha} S_{1, k}^{\alpha}
$$

where $\vec{S}^{\alpha}$ 's are $m n$-component vectors with the normalization $\sum_{\alpha}\left(\vec{S}^{\alpha}\right)^{2}=$ $m n$ where $n \rightarrow 0 ; \alpha$ goes form 1 to $m$. In this form, the Hamiltonian consists of $m$ replicas of the Hamiltonian (10), and there is one to one correspondence between the terms in the expansion of $\operatorname{Tr} e^{-H_{T}}$, and the equilibrium branched clusters. Due to presence of the additional parameter, $m$, each cluster in the expansion acquires an additional weight $m$. The partition function then reads

$$
Z_{T}=\sum_{N_{j}, N_{e}, N_{b}, N_{p}} J^{N_{b}} K^{N_{j}} h^{N_{e}} m^{N_{c}} \mathcal{N}\left(N_{j}, N_{e}, N_{b}, N_{c}\right)
$$

where $N_{j}, N_{e}, N_{b}, N_{c}$ are the number of junctions, ends, bonds, and clusters, respectively. The parameter $m$ can be analytically continued to non-integer values and has the meaning of the fugacity conjugate to the total number of clusters in the system. For self-assembled branched clusters, the relevant value is $m \rightarrow 1$, because the number of clusters is not constrained [23.

The Hamiltonian (1) can be rewritten, making the rotation in the $m$ dimensional space of $\alpha$-components, with the use of a set of $m$-dimensional orthogonal vectors $\vec{v}_{l} ; l=0, \ldots, m-1$, with $\vec{v}_{0}=\frac{1}{\sqrt{m}}(1,1, \ldots, 1)$ and $\vec{v}_{l} \cdot \vec{v}_{l^{\prime}}=$ $\delta_{l l^{\prime}}$ [23]. The new variables are then defined as:

$$
\begin{aligned}
\vec{S}^{0} & =\sum_{\alpha} \vec{S}^{\alpha} v_{0}^{\alpha}=\frac{1}{\sqrt{m}} \sum_{\alpha} \vec{S}^{\alpha} \\
\vec{S}^{l} & =\sum_{\alpha} \vec{S}^{\alpha} v_{l}^{\alpha} \quad \text { for } \quad l \neq 0
\end{aligned}
$$

Note that the normalization remains the same: $\sum_{l=0}^{m-1}\left(S^{l}\right)^{2}=m n$. The 
Hamiltonian expressed in the new variables then reads

$$
\begin{aligned}
H_{T}= & h \sqrt{m} \sum_{i} \sum_{l=0}^{m-1} S_{i}^{l}+J \sum_{\langle i, j\rangle} \sum_{l=0}^{m-1} S_{i}^{l} S_{j}^{l}+ \\
& +\frac{K}{\sqrt{m}} \sum_{\langle i, j, k\rangle}\left[S_{i}^{0} S_{j}^{0} S_{k}^{0}+\left(S_{i}^{0} \sum_{l=0}^{m-1} S_{j}^{l} S_{k}^{l}+\operatorname{perm}(i, j, k)\right)+\sum_{l \neq 0, l^{\prime} \neq 0, l^{\prime \prime} \neq 0} a_{l l^{\prime} l^{\prime \prime}} S_{i}^{l} S_{j}^{l^{\prime}} S_{k}^{l^{\prime \prime}}\right]
\end{aligned}
$$

where $a_{l l^{\prime} l^{\prime \prime}}$ are numerical coefficients, depending on the value of $m$. It can be shown then that the correlation function $\left\langle S_{i}^{0} S_{j}^{0}\right\rangle$ measures the correlation between any two endpoints and the $\left\langle S_{i}^{l} S_{j}^{l}\right\rangle$ measures the correlations between two endpoints belonging to the same cluster [23].

In the mean field approximation, the products of $S^{l}$ with $l \neq 0$ do not contribute either to the free energy or to the two point correlation function, because only $\left\langle S^{0}\right\rangle \neq 0$ (they do, however, contribute to higher order correlation functions), and can be neglected. In particular, the fact that the mean field two-point correlation function is independent of the coefficients $a_{l l^{\prime} l^{\prime \prime}}$ (i.e. , of the precise way in which weights are assigned to loops), means that the mean field approximation takes into account only the loopless graphs contributing to the two point correlation function. With this in mind, the last term in Eq.(60) vanishes, and in the limit $m \rightarrow 1$ the Hamiltonian (2) becomes formally identical to the Hamiltonian (45) of Sec. 5, with $S^{l}(l \neq 0)$ taking place of a 'transverse' component $S_{\gamma}, \gamma \neq 1$.

\section{References}

[1] P.-G. De Gennes, Scaling Concepts in Polymer Physics, Cornell Univ. Press, 1979

[2] M. Doi, Introduction to polymer physics, Clarendon Press, 1996.

[3] W.M. Gelbart, A. Ben-Shaul, D. Roux, eds., Micelles, Membranes, Microemulsions and Monolayers, Springer-Verlag, 1994

[4] P. J. Flory, Principles of Polymer Chemistry, Cornell University Press, 1981. 
[5] G. Gompper, M. Schick, Self-assembling Amphiphilic Systems (vol.16 in 'Phase transitions and critical phenomena'), Academic Press, 1994, and references therein.

[6] S.A. Safran, Statistical Thermodynamics of Surfaces, Interfaces and Membranes, (Frontiers in Physics Series), Addison-Wesley, 1994, and references therein.

[7] I.S. Gradsteyn, I.M. Ryzhik, Table of Integrals, Series and Products, Academis Press, 1980

[8] K. Huang, Statistical Mechaincs, John Wiley, 1963

[9] M. Albers et al., Molecular Biology of the Cell, Garland Publishing, 1994

[10] MRS proceedings, MRS, Pennsylvania, 2001, and references therein

[11] J.D. Jackson, Classical Electrodynamics, John Wiley \& Sons, 1975

[12] M. Daoud et al., Macromolecules 8, 804 (1975)

[13] P.-G. DeGennes,Phys. Lett, A38, 339 (1972)

[14] J. des Cloiseaux, J. Phys. (Paris) 36, 281 (1975)

[15] L. Schafer, T.A. Witten, J.Chem. Phys., 66, 2121 (1977)

[16] P.D. Gujrati, Phys. Rev. B, 25, 3381 (1982)

[17] Z.-G. Wang, M.E. Costas, W.M. Gelbart, J. Phys. Chem. 97, 1237 (1993); P. Panizza et al., J. Phys.: Cond. Matt., 10, 11659 (1998)

[18] S. P. Obukhov, J. Phys. A, 15, L211 (1982)

[19] J.C. Wheeler, P. Pfeuty, Phys. Rev. A, 24, 1050 (1991)

[20] J.C. Wheeler, P. Pfeuty, J. Chem. Phys., 74, 6415 (1981)

[21] R.G. Petschek, P. Pfeuty, J.C. Wheeler, J. Phys. (Paris), 45, L1183 (1984)

[22] In the case of 'living' self-assembled polymers, a 'polymerization' transition takes place at the very low densities, at which long chains start to be formed as opposed to a solution of monomers (cf. Ref. [20]) 
[23] T.C. Lubensky, J. Isaacson, Phys. Rev. Lett. 41, 829 (1978); T.C. Lubensky, J. Isaacson, Phys. Rev. A 20, 2130-2146 (1979)

[24] A.B. Harris et al., Phys. Rev. Lett., 35, 327 (1975); R. Kikuchi, J. Chem. Phys, 53, 2713 (1970); A. Coniglio, Phys. rev. B, 13, 2194 (1976); K.K. Murata, J. Phys. A, 12, 81 (1979)

[25] T. Miura et al., Phys. Rev. E, 54, 6596 (1996); F. Tanaka, Physica A, 257, 245 (1997); A. Moussaid, J. Phys II, 1, 637 (1991)

[26] S.K. Kumar, A. Z. Panagiotopoulos, Phys. Rev. Lett., 82, 5060 (1999) and references therein

[27] S. Panyukov, Y. Rabin, Macromolecules, 29, 7960 (1996)

[28] M.Daoud, L.Leibler, Macromolecules, 21, 1497 (1988)

[29] A.N. Semenov, M. Rubinstein, 31, 1373 (1988)

[30] A.H. Narten et al.,J. Chem. Soc. Faraday Trans, 88, 1791 (1992)

[31] G. Cristobal et al., Physica A, 268, 50 (1999)

[32] K. Elleuch et al., J. Phys I, 5, 465 (1995)

[33] A. Khatory et al., Langmuir, 9, 933 (1993); E. Buhler et al., J. Phys II, 5, $765(1995)$

[34] A. Berheim-Groswasser et al., Langmuir, 15: 5448 (1999); A. BerheimGroswasser et al., Langmuir, 16, 4131 (2000)

[35] T. Tanaka et al., Phys. Rev. Lett, 42, 1556 (1979)

[36] F. Tanaka, Physica A, 257, 245 (1998), F. Tanaka, Macromolecules, 31, 384 (1998)

[37] K.S. Schweizer, J.G. Curro, Macromolecules, 21, 3070 (1988); Macromolecules, 21, 3082 (1988); K.S. Schweizer et al., Macromolecules, 28, $1528(1995)$

[38] T. A. Vilgis, Physics Reports, 336, 167 (2000)

[39] R. Bidaux et al., J. Stat. Phys., 45, 113 (1986) 
[40] S.T. Milner et al., J. Phys (France), 49, 1065 (1988)

[41] T. Drye, M.E. Cates, J. Chem. Phys. 96 (2), 1367 (1992)

[42] T. Tlusty T, S.A. Safran, R. Strey, Phys. Rev. Lett., 84, 1244 (2000);T. Tlusty, S.A. Safran, J. Phys. Cond. Matt., 12, A253 (2000)

[43] R. Strey et al., J. Chem. Phys, 105, 1175 (1996); F. Lichterfeld et al, J. Phys. Chem., 90, 5762 (1986) and references therein

[44] P.G. DeGennes, C. Taupin, J. Phys. Chem., 86, 2294 (1982)

[45] T. Tlusty, S.A. Safran, Science, 290, 1328 (2000)

[46] P. G. de Gennes and P. A. Pincus, Phys. Kondens. Mater. 11, 189 (1970)

[47] D. Andelman et al., J. Chem. Phys., 87 (12), 7229, (1987); M.E. Cates, et al, Europhys. Lett., 5, 7339 (1988). S.T. Milner et al, J. Phys. (France), 49. 1065 (1988)

[48] M. Tempel, G. Isenberg, E. Sackmann, Phys. Rev. E, 54, 1802 (1996). 
This figure "corr.jpg" is available in "jpg" format from: http://arxiv.org/ps/cond-mat/0205488v1 
This figure "hex.jpg" is available in "jpg" format from: http://arxiv.org/ps/cond-mat/0205488v1 
This figure "junclat.jpg" is available in "jpg" format from: http://arxiv.org/ps/cond-mat/0205488v1 
This figure "netw.jpg" is available in "jpg" format from: http://arxiv.org/ps/cond-mat/0205488v1 
This figure "onejunction.jpg" is available in "jpg" format from: http://arxiv.org/ps/cond-mat/0205488v1 
This figure "peak.jpg" is available in "jpg" format from: http://arxiv.org/ps/cond-mat/0205488v1 
This figure "phd1flex.jpg" is available in "jpg" format from: http://arxiv.org/ps/cond-mat/0205488v1 
This figure "translong.jpg" is available in "jpg" format from: http://arxiv.org/ps/cond-mat/0205488v1 